\title{
Immunogenicity and safety of routine vaccines in children and adolescents with rheumatic diseases on immunosuppressive treatment - a systematic review
}

\author{
Michèle Keller ${ }^{1} \cdot$ Laure F. Pittet $^{2,3,4}$ ([) Petra Zimmermann ${ }^{1,3,5}$
}

Received: 11 April 2021 / Revised: 25 September 2021 / Accepted: 3 October 2021 / Published online: 22 December 2021

(c) The Author(s) 2021

\begin{abstract}
The immunogenicity of vaccines in children with juvenile autoimmune rheumatic diseases (JARDs) can be reduced, there are additional safety concerns around vaccination, and there is a potential for worsening in disease activity. In this systematic review, we summarise studies that investigated the immunogenicity and safety of routine vaccines in children and adolescents with JARD on immunosuppressive treatment. We identified 37 studies investigating 2571 children and adolescents with JARD on immunosuppressive treatment and 4895 control children. Of the 56 geometric mean antibody titres measured, 19 (34\%) were lower, six (11\%) higher, and 31 (55\%) similar; of the 39 seroprotection rates measured, 10 (26\%) were lower, two (5\%) higher, and 27 (69\%) similar; and of the 27 seroconversion rates measured, nine (33\%) were lower, two (8\%) higher, and $16(59 \%)$ similar in children with JARD on immunosuppressive treatment compared with control children. However, many of the studies were underpowered, and not designed to show non-inferiority between children with JARD and controls. Subgroup analysis for different types of immunosuppressive treatments was not feasible, as most studies did not report results by treatment. Severe adverse events were reported in 38 children ( 33 with juvenile idiopathic arthritis, four with systemic lupus erythematosus, and one in a healthy child); most of them were likely not related to the vaccination (e.g. elective hospitalisation or surgery). A worsening in disease activity was reported in 44 (2\%) children with JARD; again, many of them were likely not related to the vaccination. There were no safety concerns with live attenuated vaccines; however, only few studies reported results for this. Conclusion: Vaccination in children with JARD on immunosuppressive treatment is safe and should be promoted, especially since these children are at increased risk for infection. The importance for the completion of vaccination schedules should be stressed. Strategies to compensate for the lower vaccine responses, which are found in approximately one-third of these children, include measuring antibody levels to determine the optimal timing for the administration of additional booster doses.
\end{abstract}

\section{What is Known:}

- Children with juvenile autoimmune rheumatic diseases (JARDs) are at higher risk for infections, due to their underlying disease and their immunosuppressive treatment.

- In children with JARD, the immunogenicity of vaccines might be reduced, and concerns about safety or the potential for worsening in disease activity after vaccination exist.

What is New:

- Our systematic review shows that vaccines in children with JARDs on immunosuppressive treatment are safe and immunogenic.

- There are several limitations of the currently published studies, including random timing of measuring vaccine responses and age differences between children with JARD and control groups. Many of the studies were underpowered, and not designed to show non-inferiority between children with JARD and controls.

Communicated by Nicole Ritz

Petra Zimmermann

petra.zimmermann@unifr.ch

Extended author information available on the last page of the article 
Keywords Antibodies - Disease activity · DMARD .

Humoral response · Immunisation · Juvenile arthritis ·

Juvenile dermatomyositis - Juvenile autoimmune rheumatic diseases $\cdot$ Systemic lupus erythematosus

$\begin{array}{ll}\text { Abbreviations } \\ \text { ERA } & \text { Enthesitis-related arthritis } \\ \text { GMT } & \text { Geometric mean titre } \\ \text { HAV } & \text { Hepatitis A virus } \\ \text { HBV } & \text { Hepatitis B virus } \\ \text { HPV } & \text { Human papilloma virus } \\ \text { JARD } & \text { Juvenile autoimmune rheumatic disease } \\ \text { JDM } & \text { Juvenile dermatomyositis } \\ \text { JIA } & \text { Juvenile idiopathic arthritis } \\ \text { MenC } & \text { Meningococcus C } \\ \text { MMR } & \text { Measles-mumps-rubella } \\ \text { MTX } & \text { Methotrexate } \\ \text { PCV7 } & \text { 7-valent pneumococcal conjugated vaccine } \\ \text { PPV23 } & \text { 23-valent pneumococcal polysaccharide vaccine } \\ \text { RCT } & \text { Randomised controlled trial } \\ \text { SAE } & \text { Severe adverse event } \\ \text { SCR } & \text { Seroconversion rate } \\ \text { SLE } & \text { Systemic lupus erythematosus } \\ \text { SPR } & \text { Seroprotection rate } \\ \text { TIV } & \text { Trivalent influenza vaccine } \\ \text { VZV } & \text { Varicella }\end{array}$

\section{Introduction}

Juvenile autoimmune rheumatic diseases (JARDs) are frequent in children and adolescents. The global incidence of juvenile idiopathic arthritis (JIA), for example, is estimated at 1 per 1000 children and has been reported to be increasing over the past decades [1,2]. Children with JARD are at higher risk for infection due to their underlying disease but also because they are often on immunosuppressive treatment. Vaccination is the most effective and economic method of preventing infectious diseases [3]. Children with JARD are often under-vaccinated and therefore at higher risk for vaccine-preventable diseases, as parents and paediatricians may refuse or delay vaccinations due to safety concerns $[4,5]$. Furthermore, compared to healthy children, the immunogenicity of vaccines in children and adolescents with JARD can be reduced and there might be concerns for a potential in worsening in disease activity $[6,7]$.

The immunogenicity and safety of vaccines in children with JARD have previously been reviewed $[8,9]$. However, in the past decade, new immunosuppressive agents have become available for the treatment of JARD in children and additional studies have been published on the immunogenicity of vaccines in this group. Therefore, there is a need of an updated overview on this topic to assure the safe and most beneficial use of vaccines in these children.
In this systematic review, we summarise studies that have investigated the immunogenicity (humoral responses) and safety of vaccines in children and adolescents with JARD on immunosuppressive treatment.

\section{Systematic review methods}

A systematic search was done according to the preferred reporting items for systematic reviews and meta-analyses, the PRISMA guidelines [10]. In March 2021, MEDLINE (1946 to present) and Embase (1947 to present) were searched using the Ovid interface with the following search term combination: "child" AND "vaccination" AND "immunosuppressive treatment" (see supplementary data for detailed search terms). No language limitations were used. We included original studies which investigated the immunogenicity (specific immunoglobulin $\mathrm{G}$ responses) and safety of routine vaccines in children and adolescents up to the age of 21 years with JARD on immunosuppressive treatment. Exclusion criteria were studies which (i) did not specify the immunosuppressive treatment, (ii) included children with renal insufficiency or on dialysis, (iii) had less than 10 participants, and (iv) did not report results for children separately to those from adults. References of retrieved articles were hand-searched for additional publications.

The following variables were extracted from the included studies: author, publication year, country, study type, level of evidence, number of participants, age and gender of participants, immunosuppressive treatment, vaccine type, vaccine brand, vaccine producer, vaccine dose, number of vaccine doses, interval between doses, timing of blood sampling after last vaccination, antibody responses, safety (including local and systemic reactions, serious adverse events and worsening in disease activity), and additional important findings. The ROBINS-1 tool was used to assess risk of bias [11].

\section{Systematic review results}

Our search identified 3488 studies. Of these, 30 fulfilled the inclusion criteria [12-41]. Seven additional studies were found by hand-searching of references [42-48]. The selection of included studies is summarised in Fig. 1. The 37 studies ( 28 cohort studies, three case-control studies, three cross-sectional studies, two randomised controlled trials (RCTs) and one case series) included in this review investigated 2571 children and adolescents with JARD on immunosuppressive treatment and 4895 control children (4865 healthy children and 30 children with non-rheumatic diseases). The number of participants in each study ranged from 23 to 2576 (median 77, mean 202). Antibodies against 22 different antigens were measured. Of the studies, 30 evaluated the safety of vaccines, 25 local reactions, 26 systemic 
reactions, 27 severe adverse events (SAEs), and 26 worsening in disease activity. All of the studies were done in industrialised countries: Brazil 11, Netherlands 8, Greece 4, Turkey 3, Germany 2, Japan 2, Iran 1, Italy 1, Slovenia 1, Spain 1, Sweden 1, Switzerland 1, and USA 1. The results of these studies are summarised in Tables 1 and 2 [12-48]. The risk of bias summary of studies included in the review can be found in Table 3.

\section{Results}

Overall, 56 geometric mean antibody titres (GMTs) were measured in children with JARD on immunosuppressive treatment, of which 19 (34\%) were lower, six (11\%) higher, and $31(55 \%)$ similar in these children compared to control children [12-16, 18-26, 28-34, 36-40, 43-45, 47, 48]. Of the 39 seroprotection rates (SPRs) measured, 10 (26\%) were lower, two (5\%) higher, and $27(69 \%)$ similar in the two groups $[12,13,16,18,19,21-23,29-35,38,39,42$,
43, 46]. Of the 27 seroconversion rates (SCRs) measured, nine $(33 \%)$ were lower, two (8\%) higher, and $16(59 \%)$ similar in children with JARD compared with control children $[12,13,18,19,21-23,28,29,31-33,37,46]$.

\section{Enthesitis-related arthritis}

\section{Measles, rubella}

One study investigated the persistence of specific antibodies after two doses of measles and rubella vaccination in 41 children with enthesitis-related arthritis (ERA) 1 and 3 years after the initiation of adalimumab and compared it with 149 healthy children [43]. At both timepoints, the GMTs were lower in children with ERA for measles and rubella compared with healthy children of similar age, while there was no difference in SPRs. No difference in GMTs or SPRs was found between children on adalimumab only and children who were on additional methotrexate (MTX) or sulfasalazine. The study provided no information about the safety of the vaccines.

Fig. 1 Selection of studies
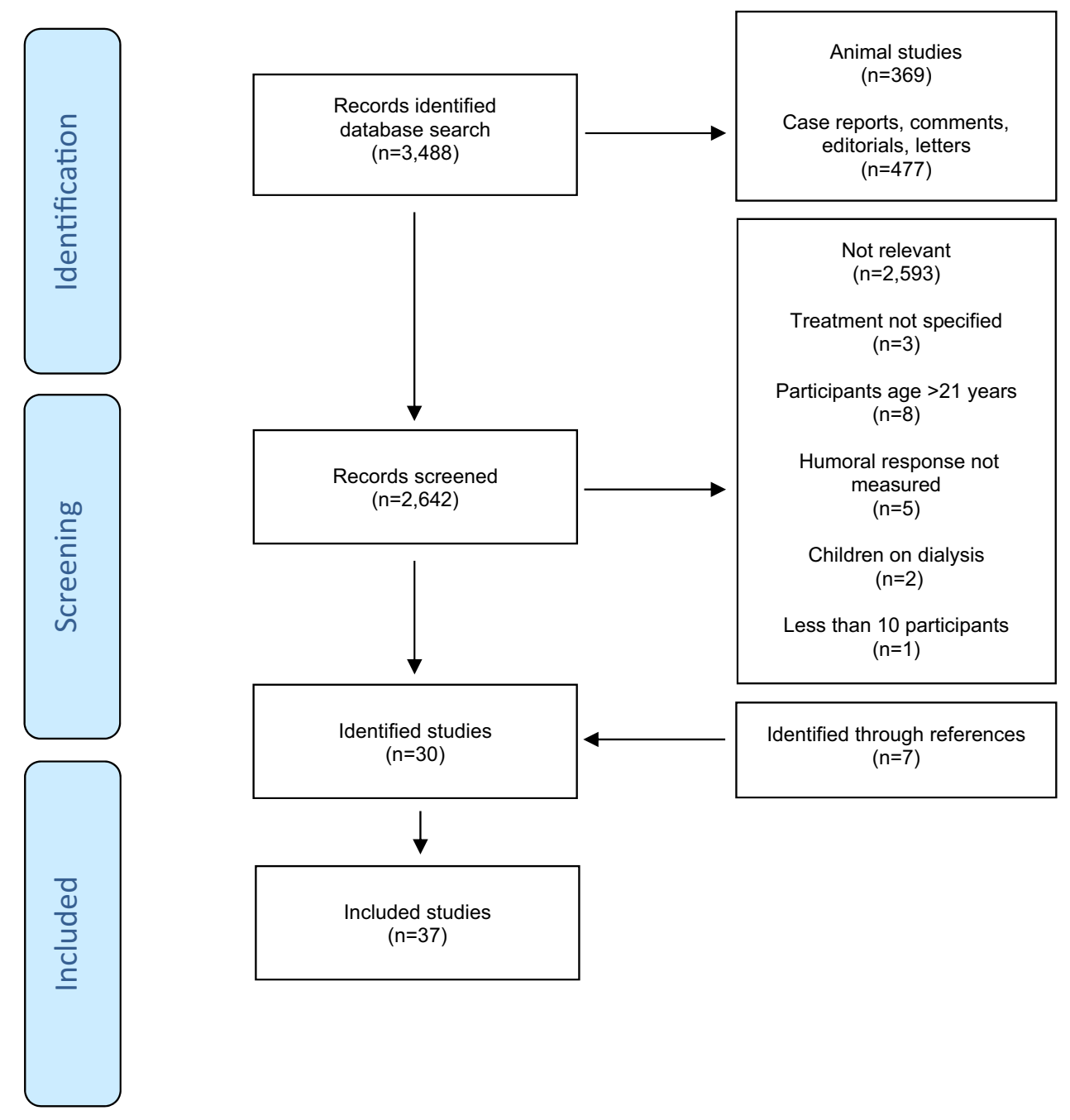


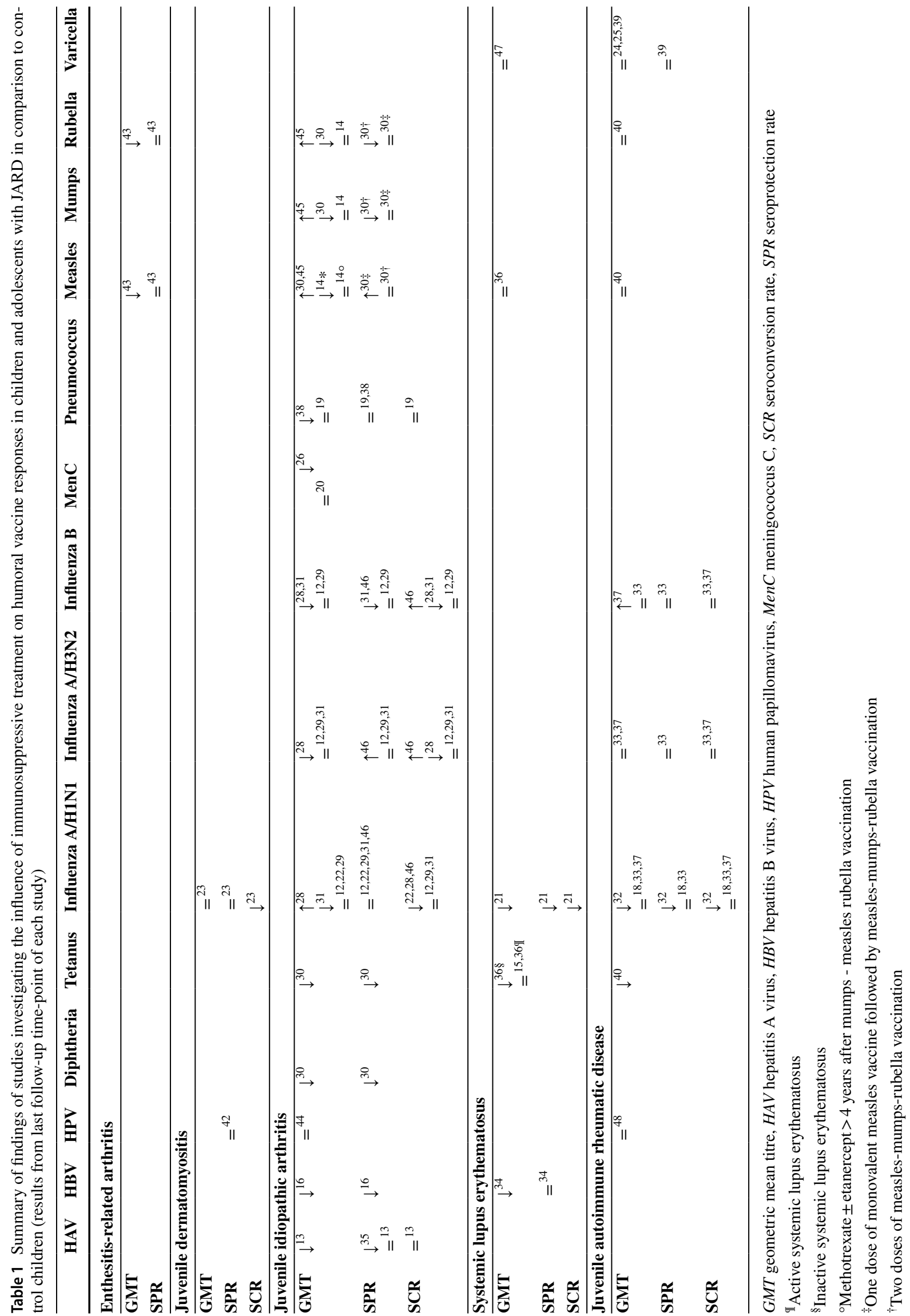




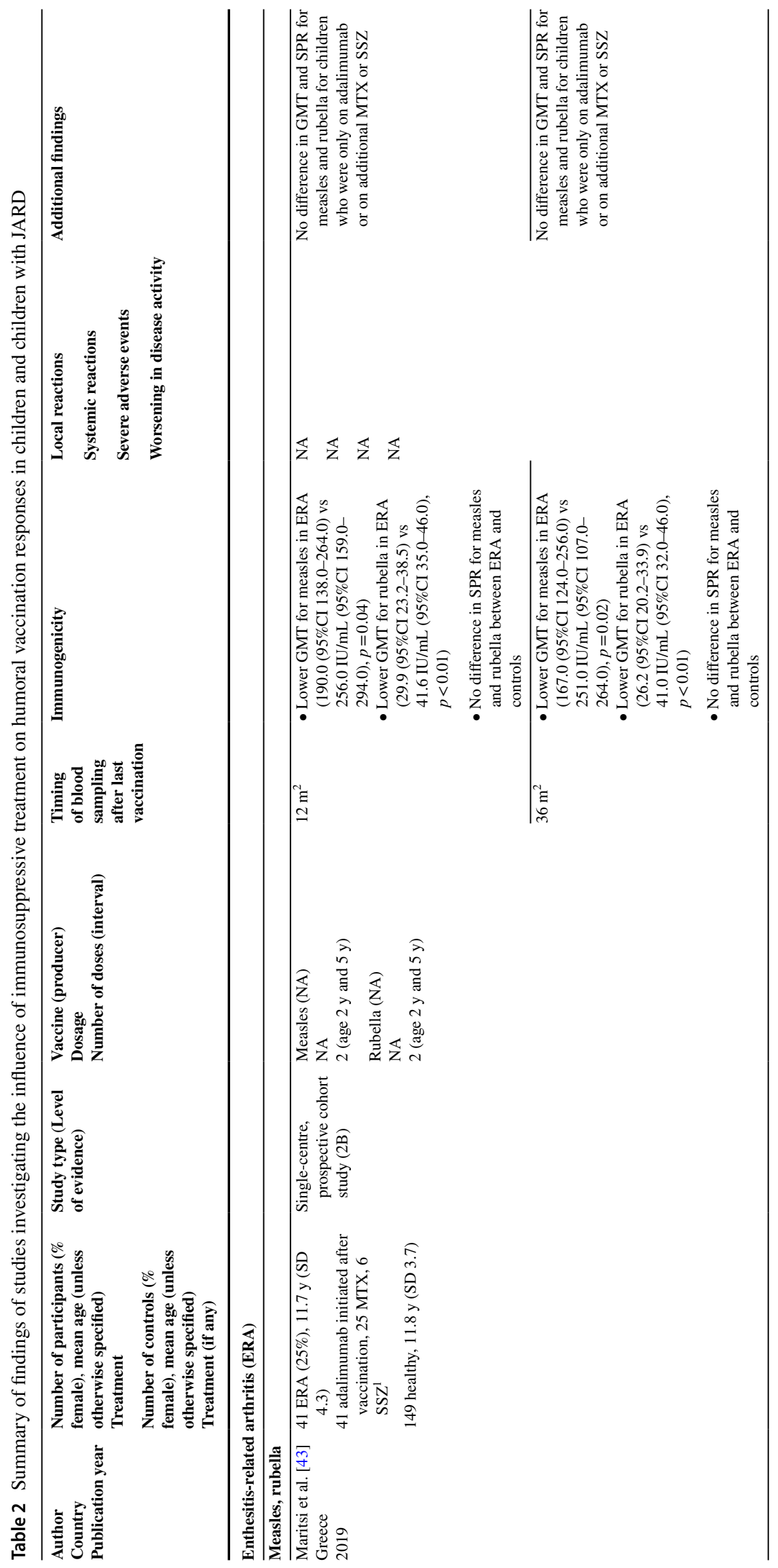




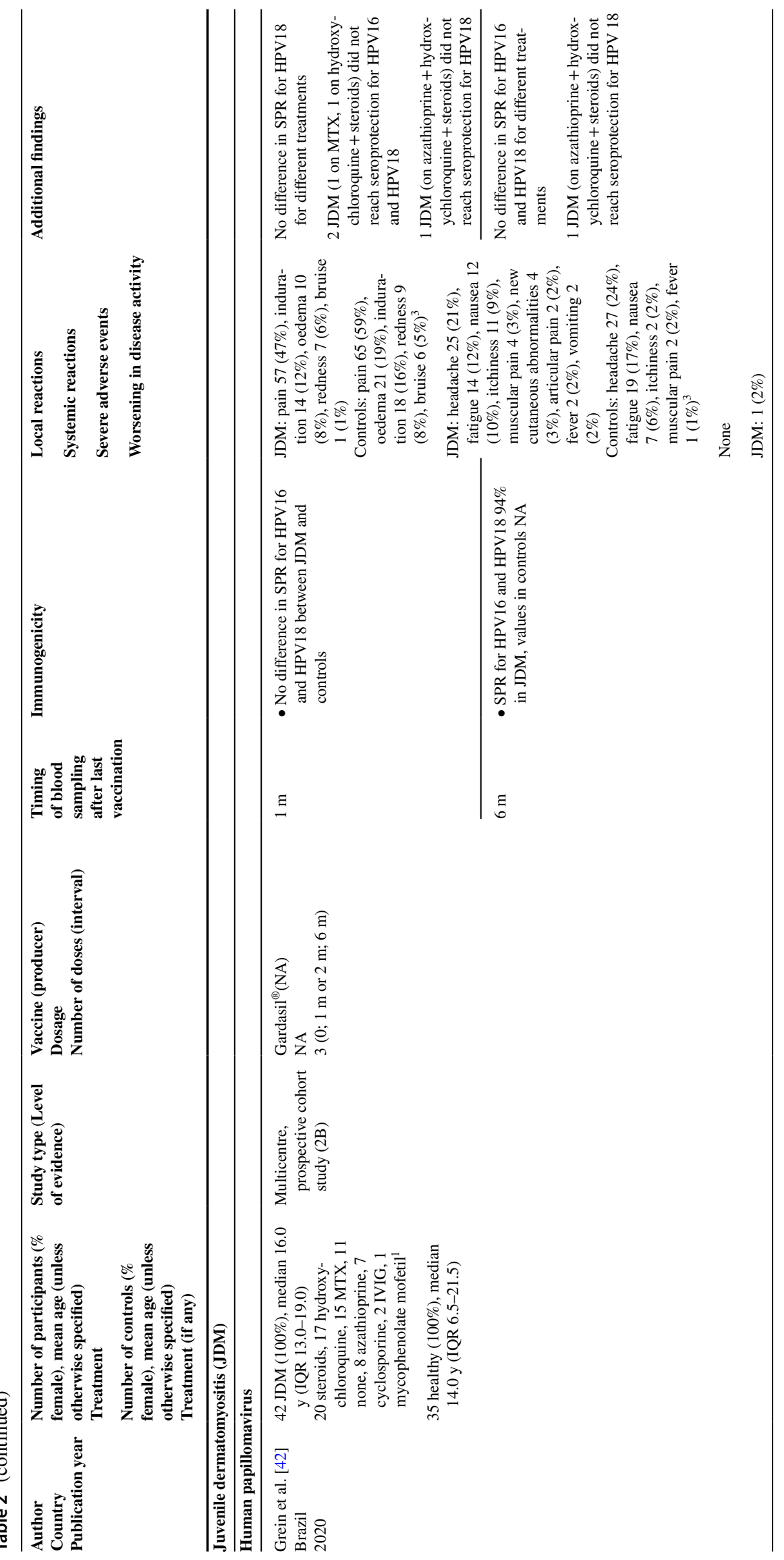

Springer 


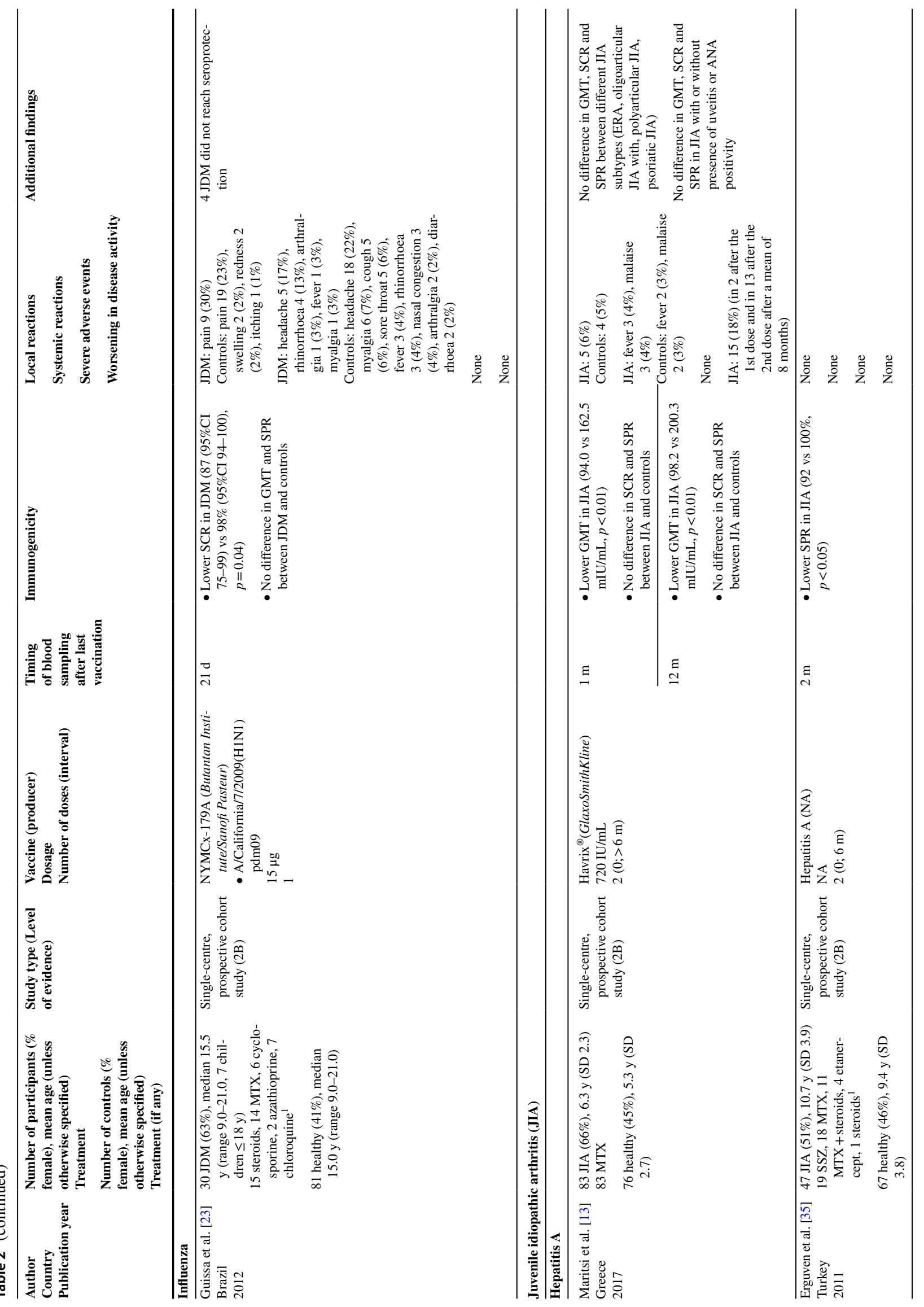




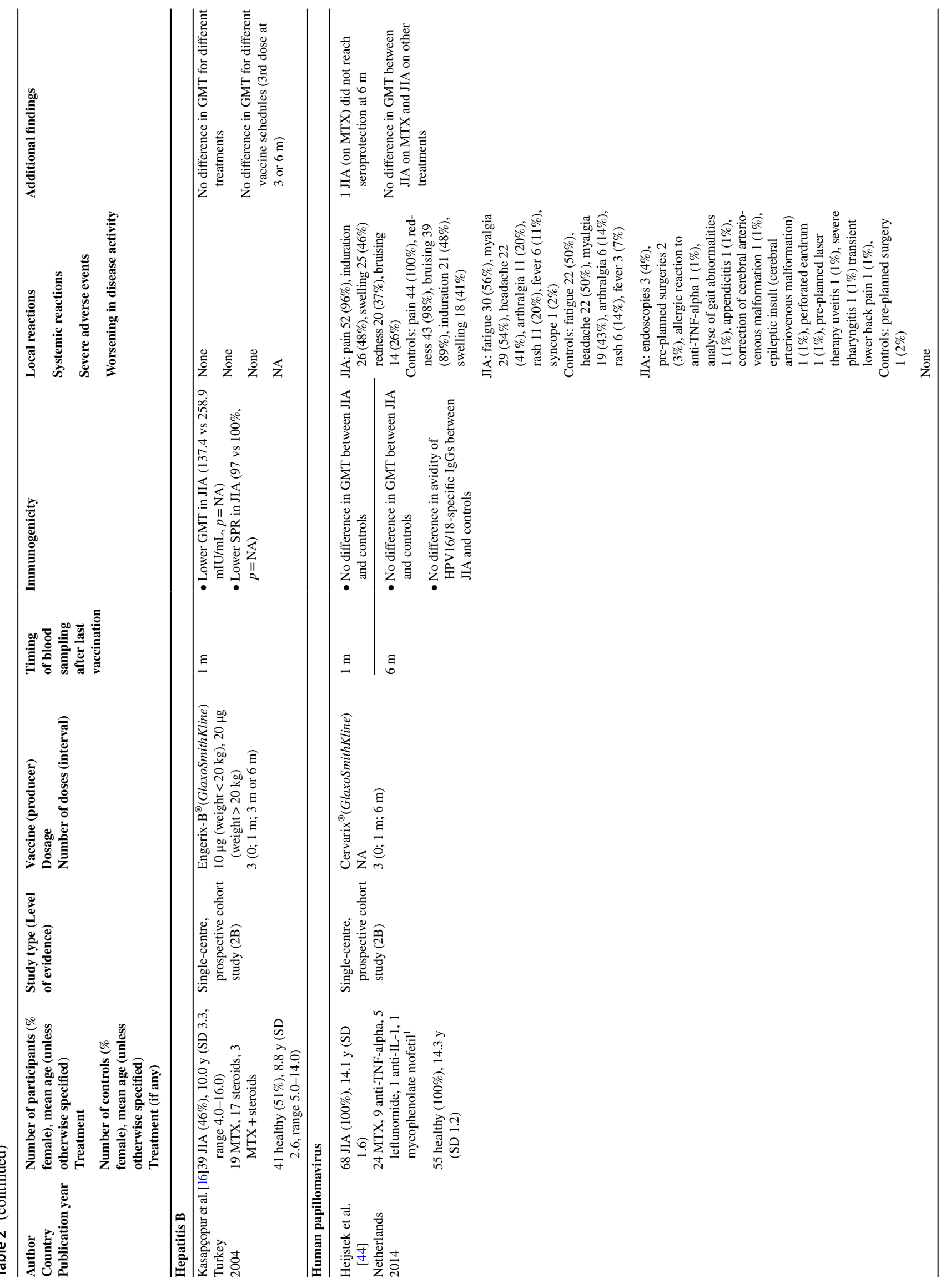




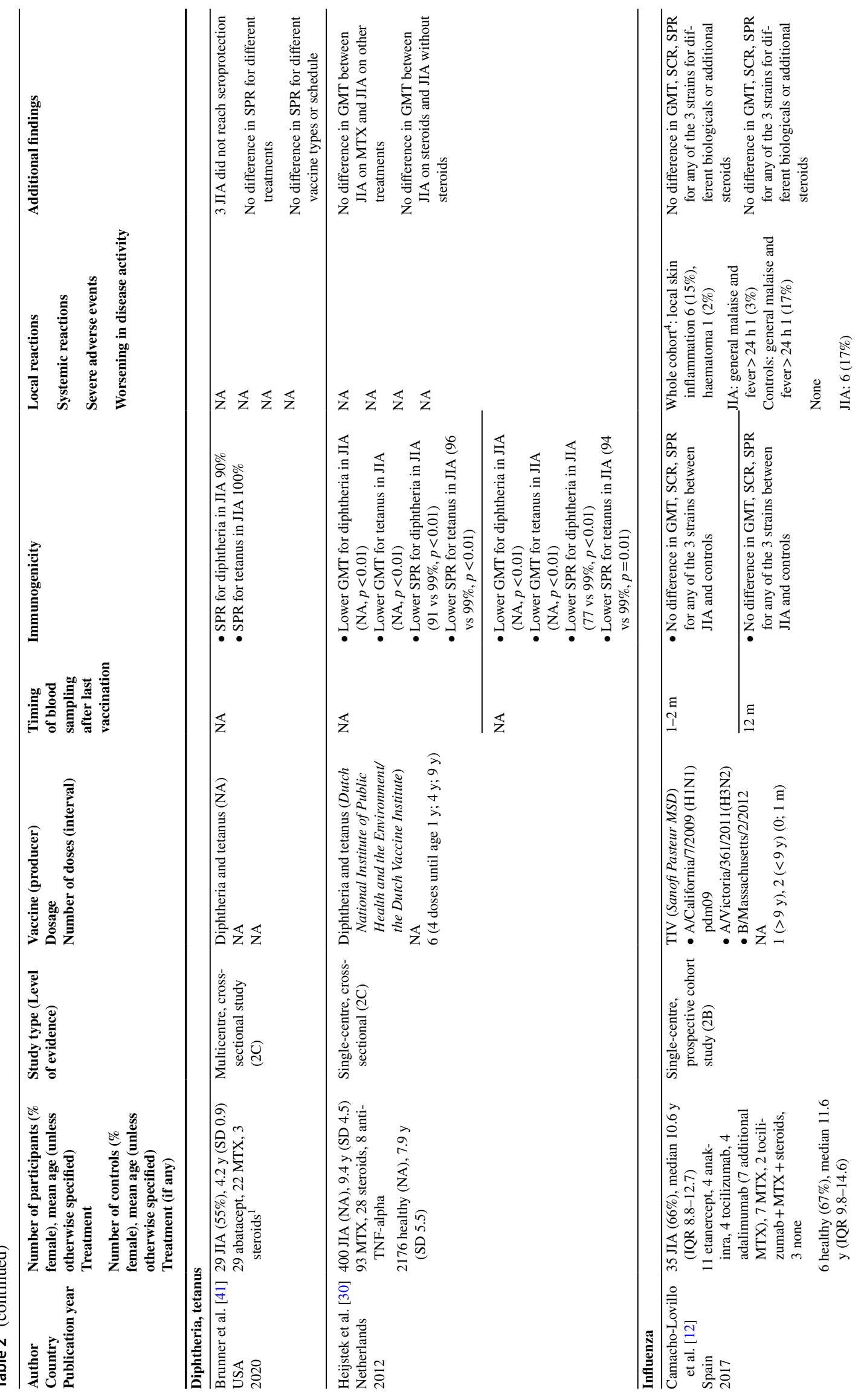




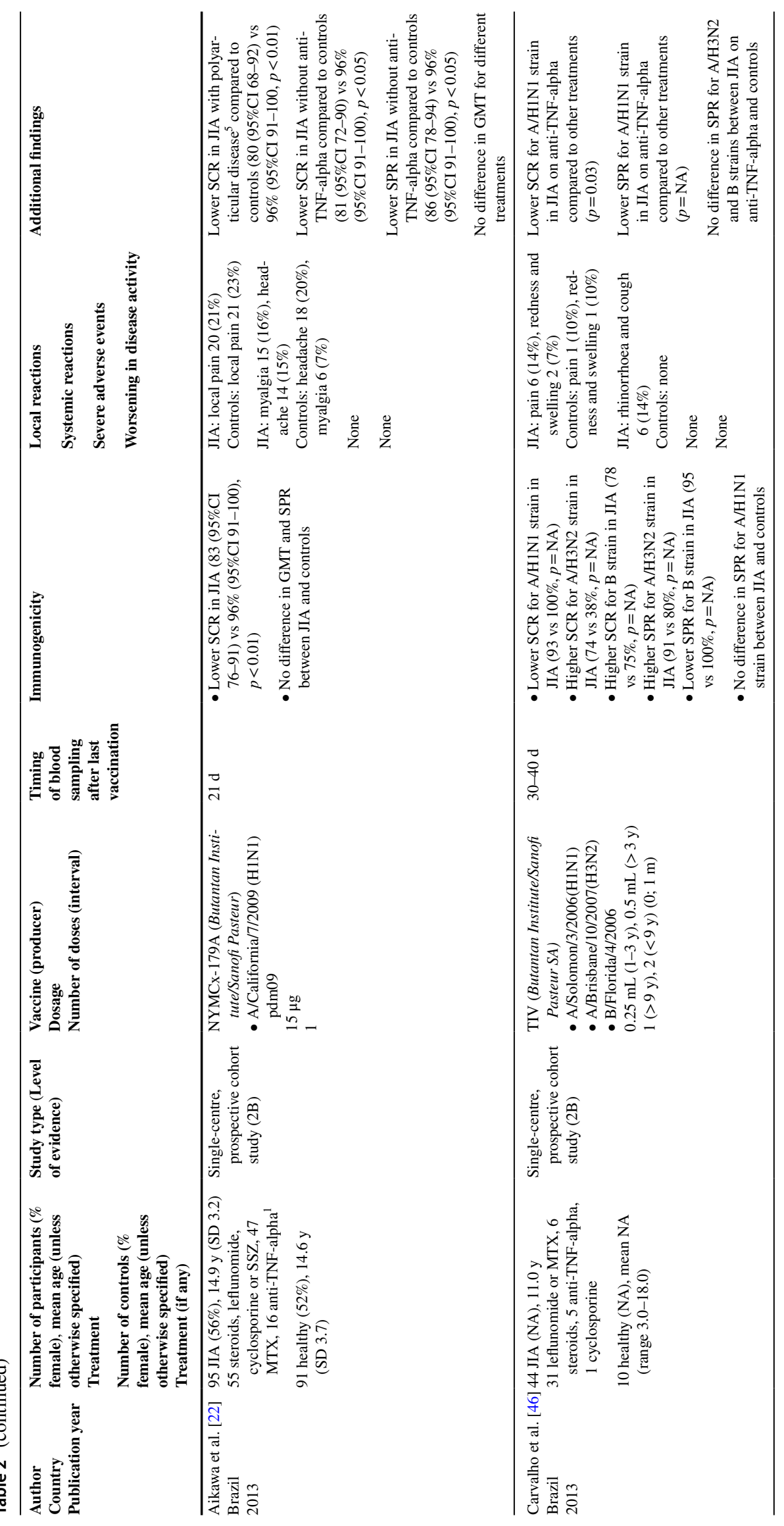

Springer 


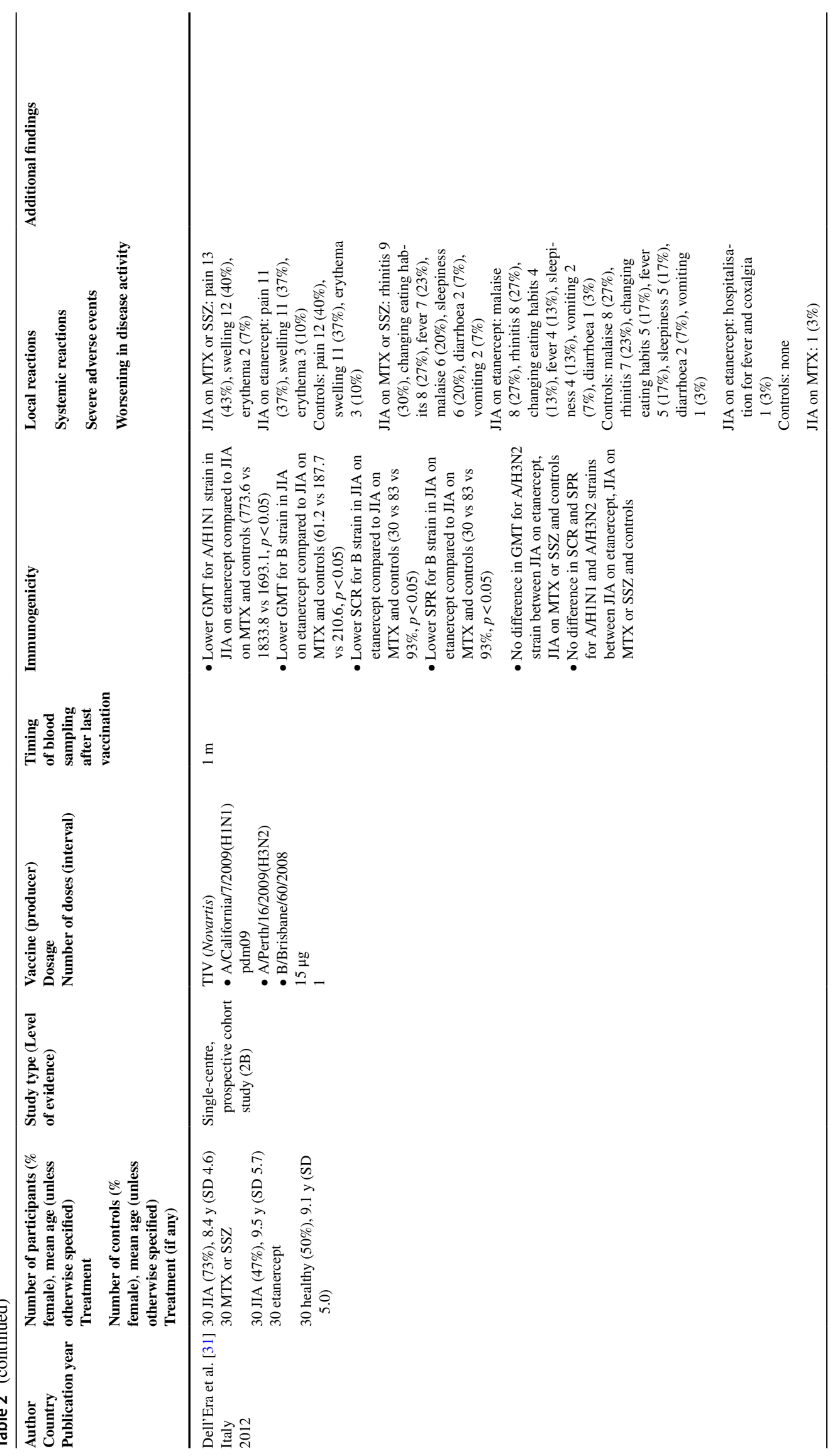




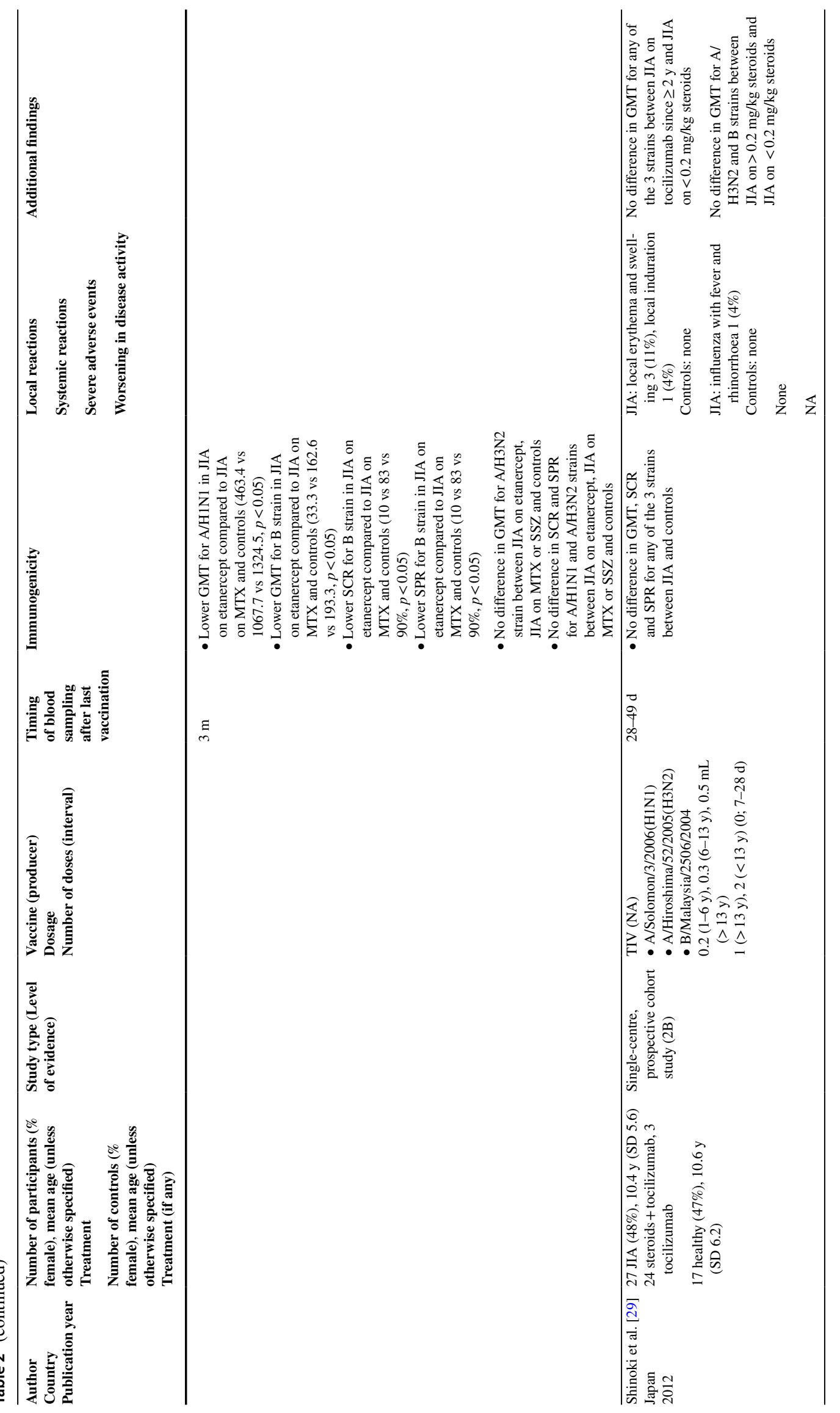

Springer 


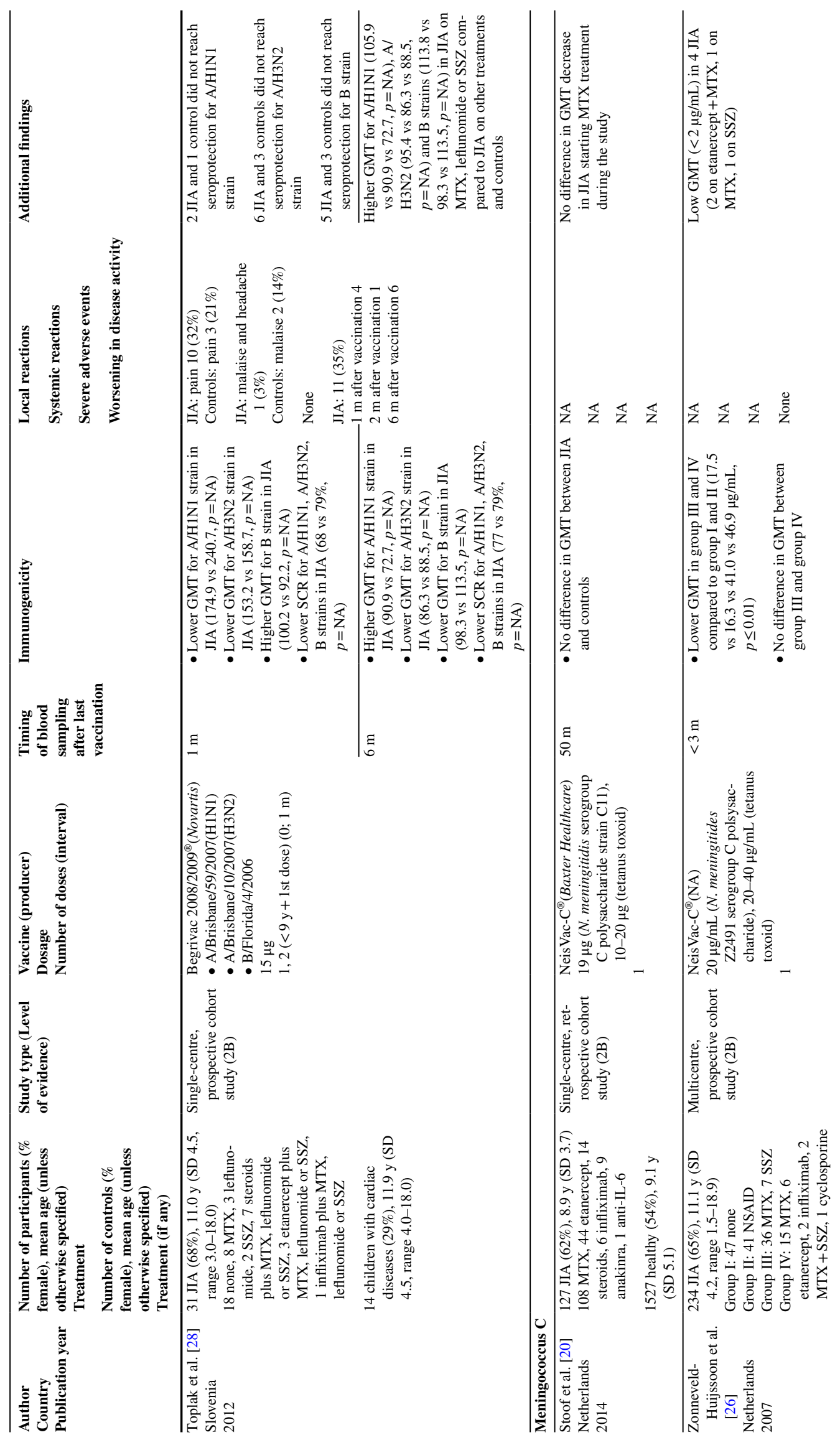




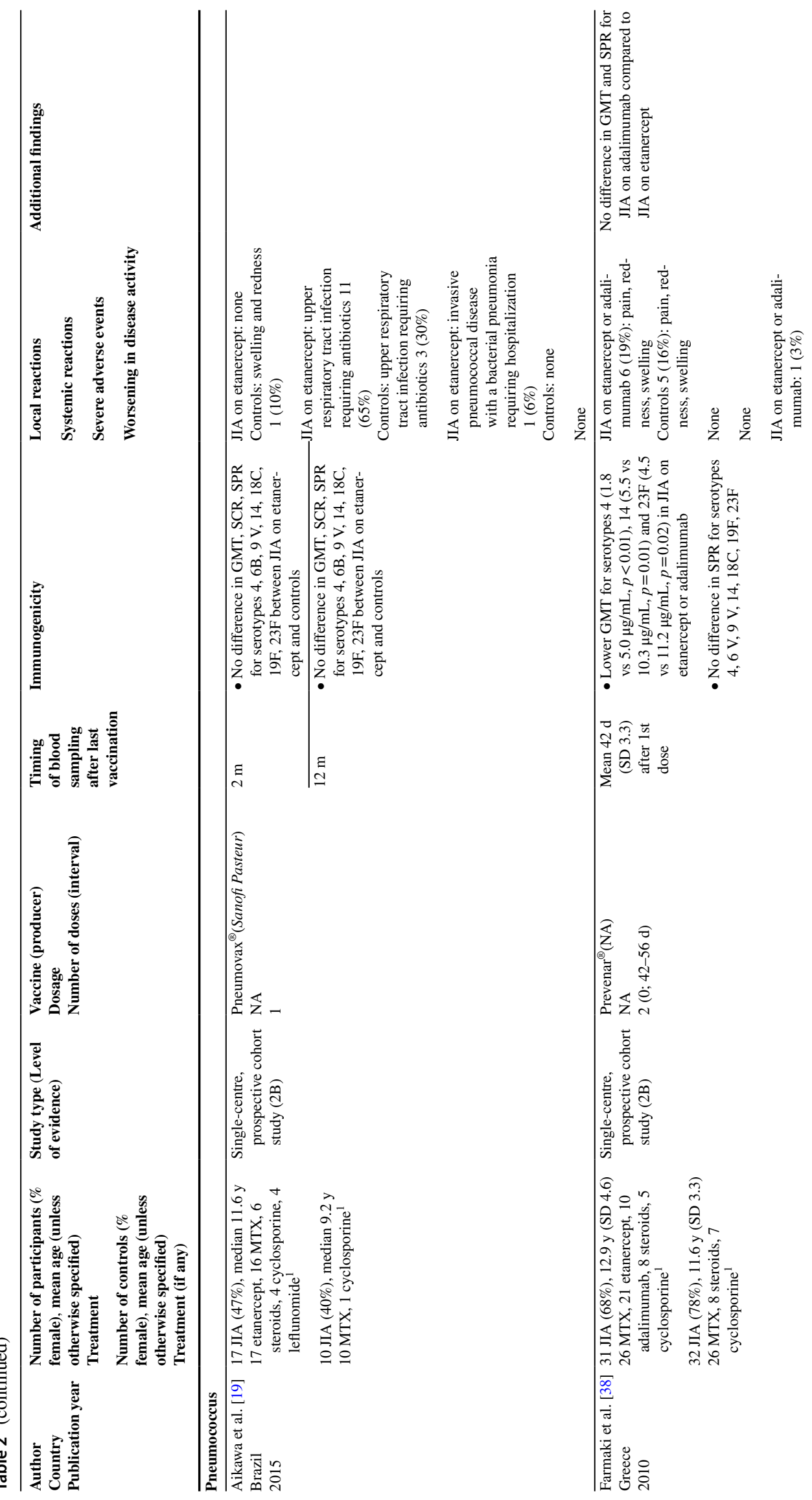




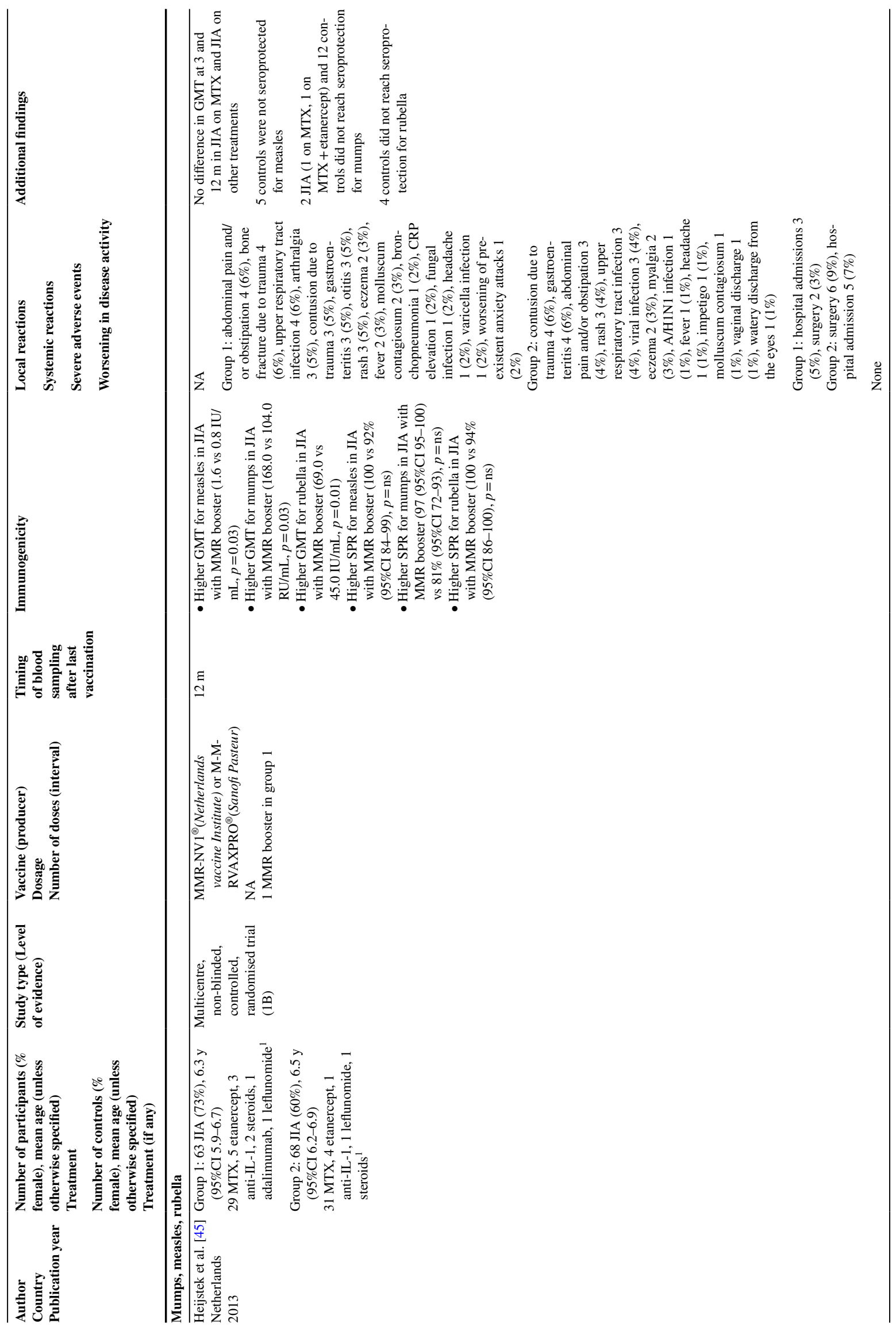




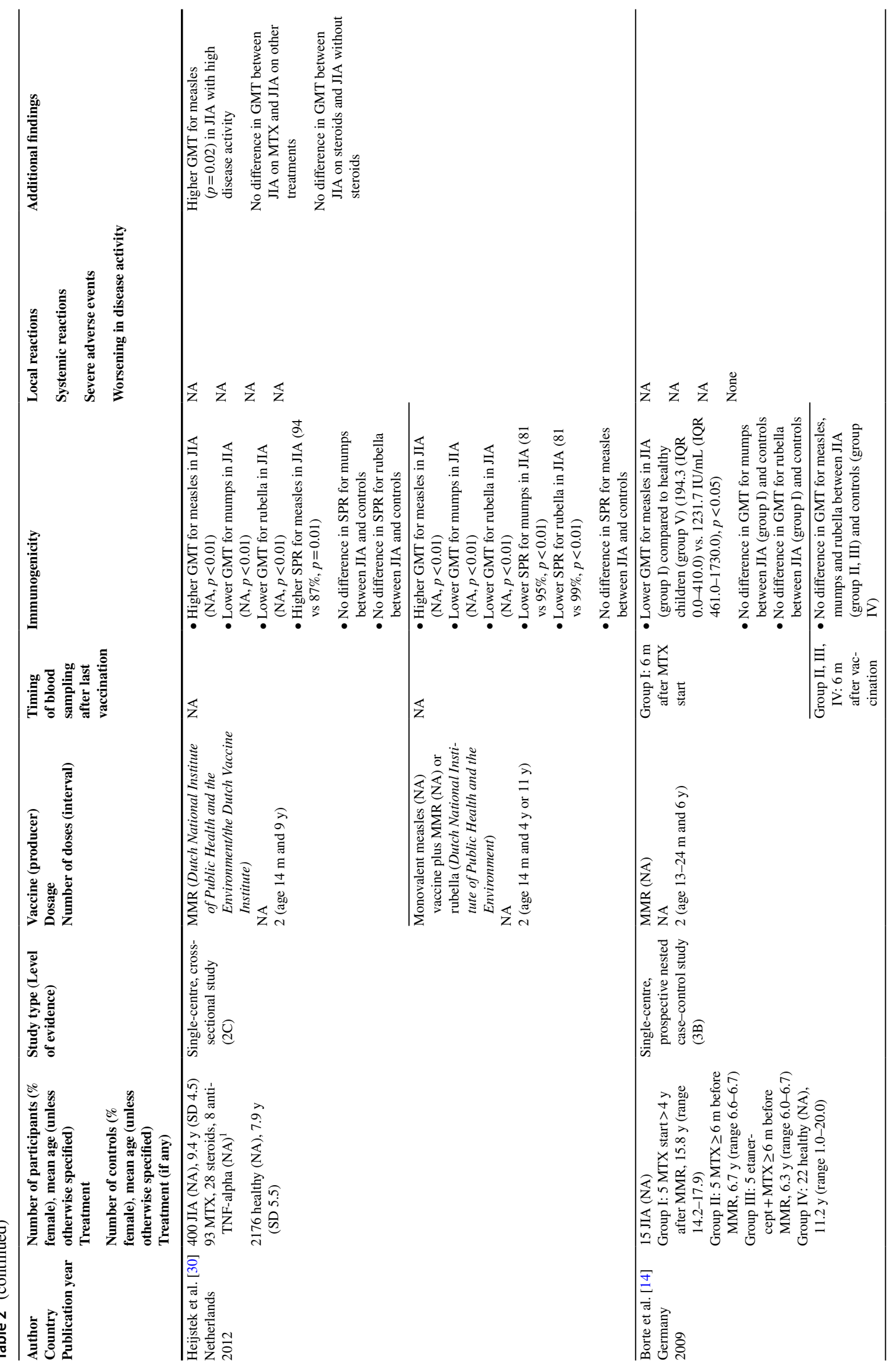




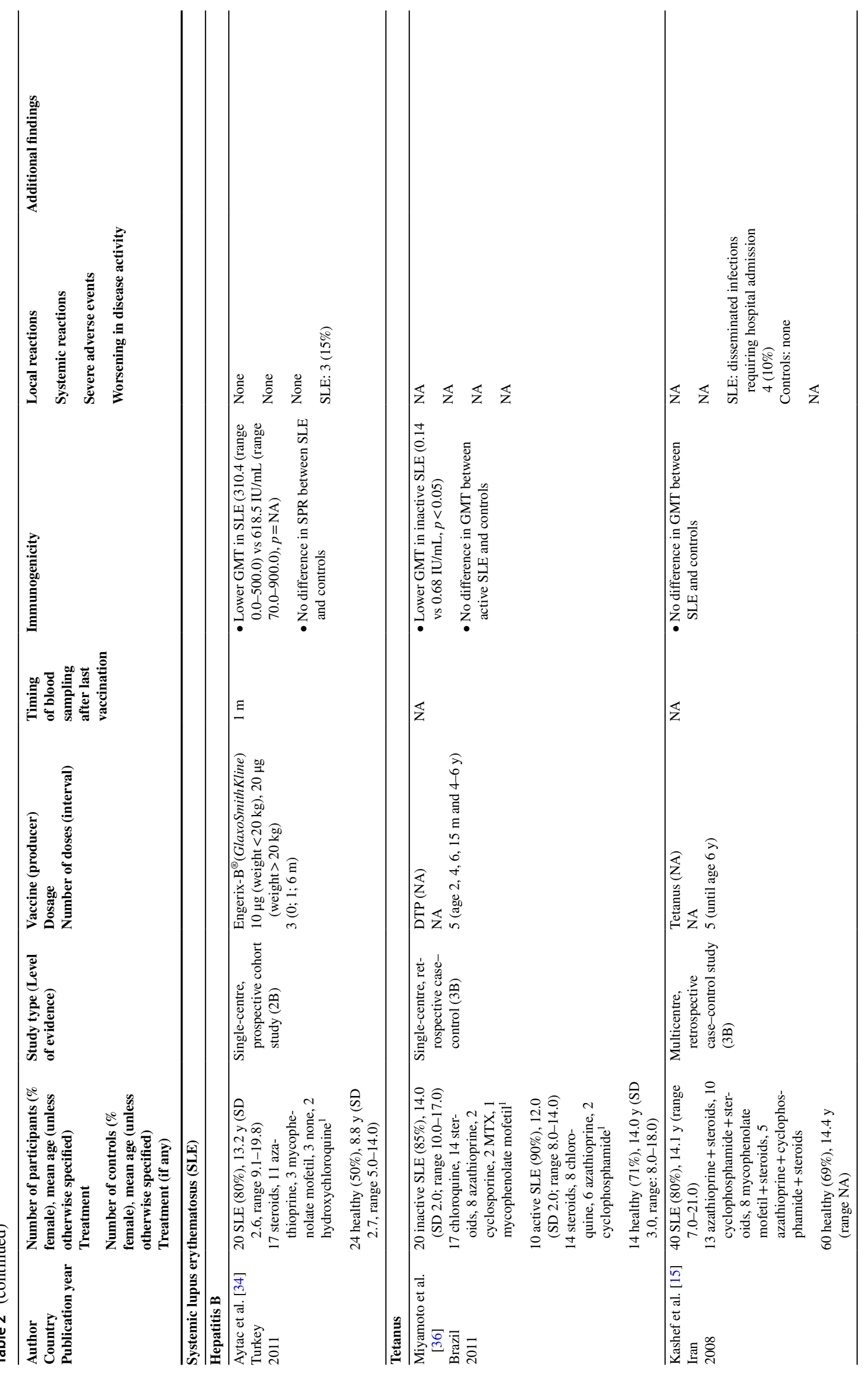




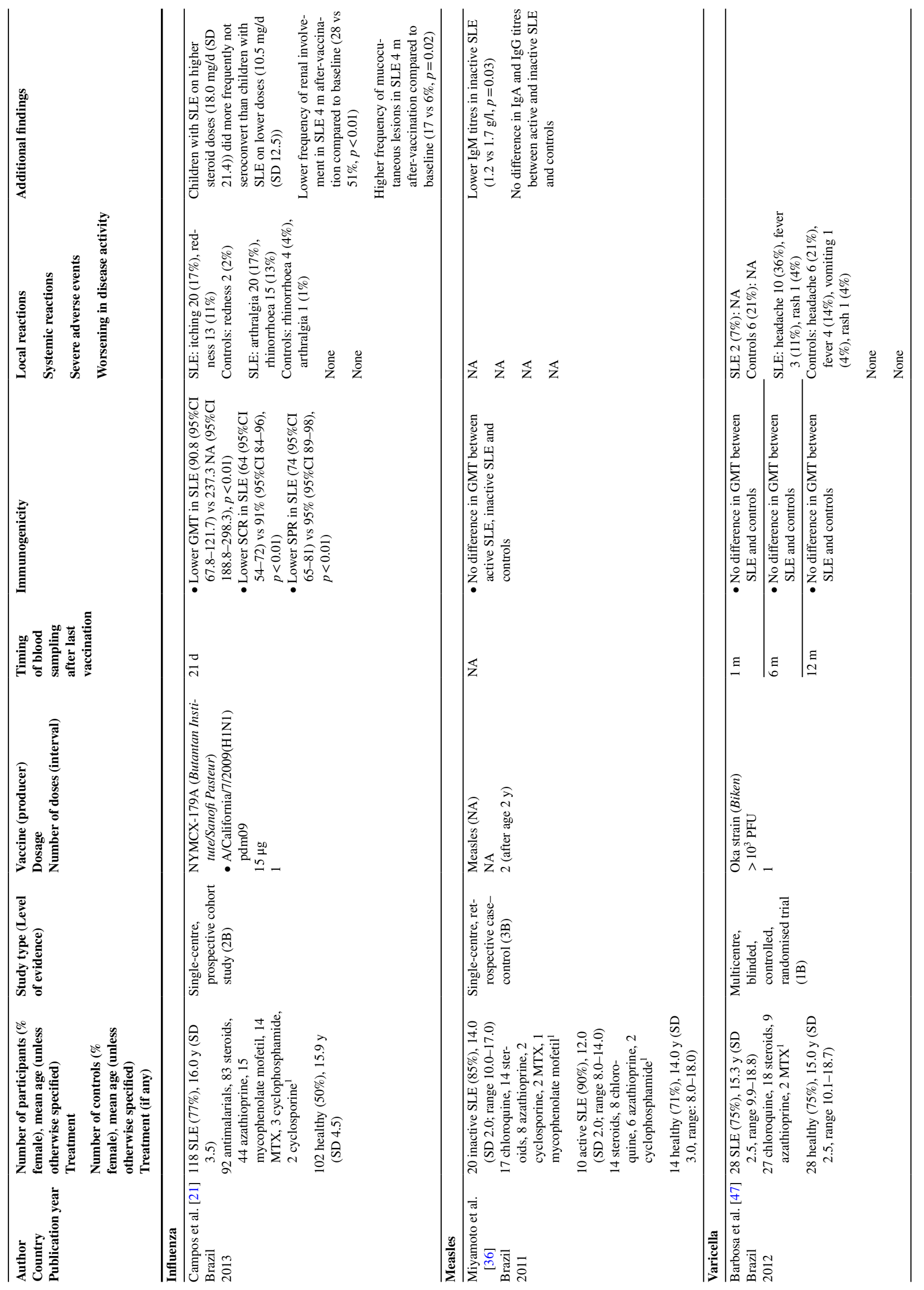




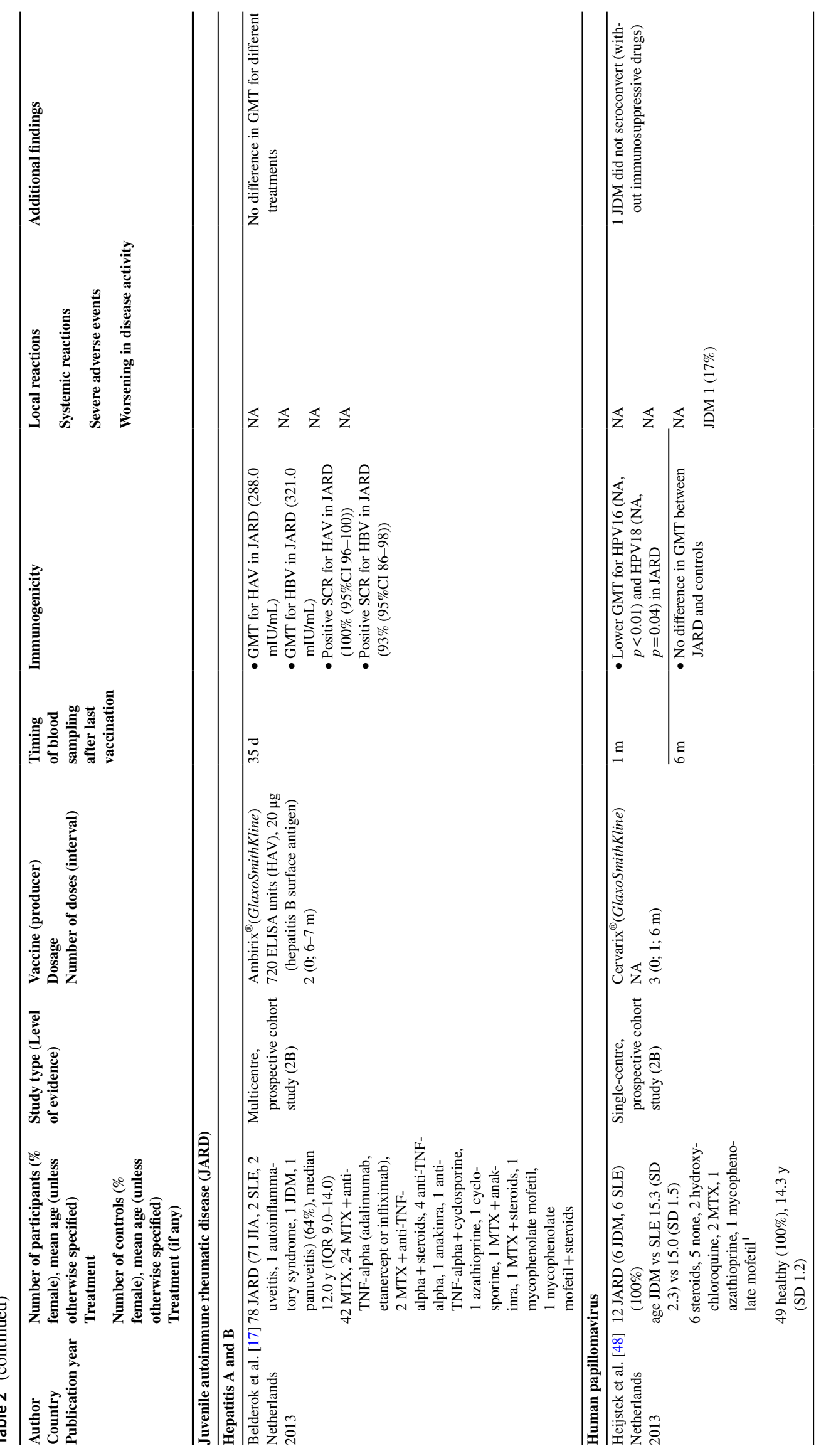




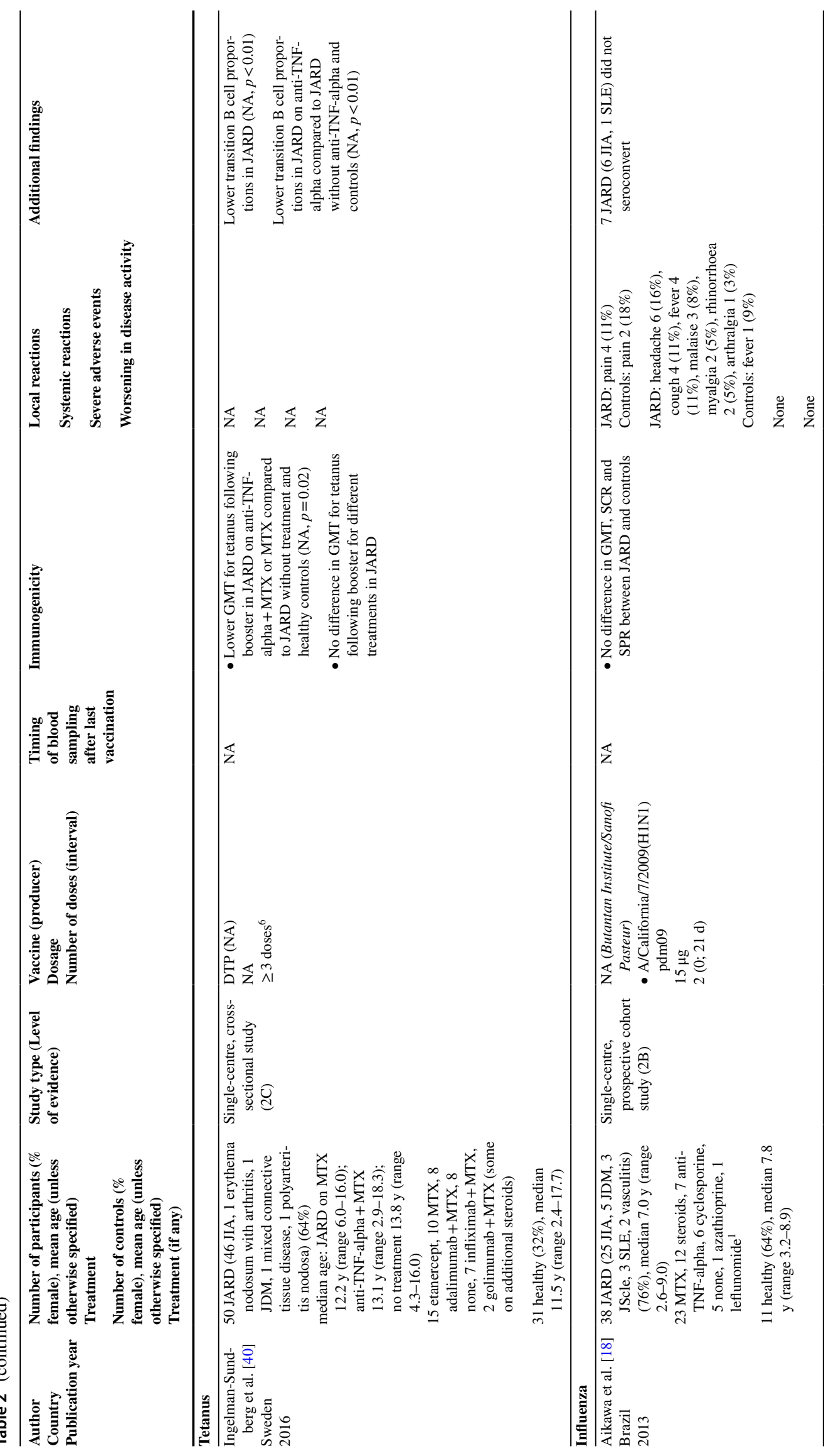

Springer 


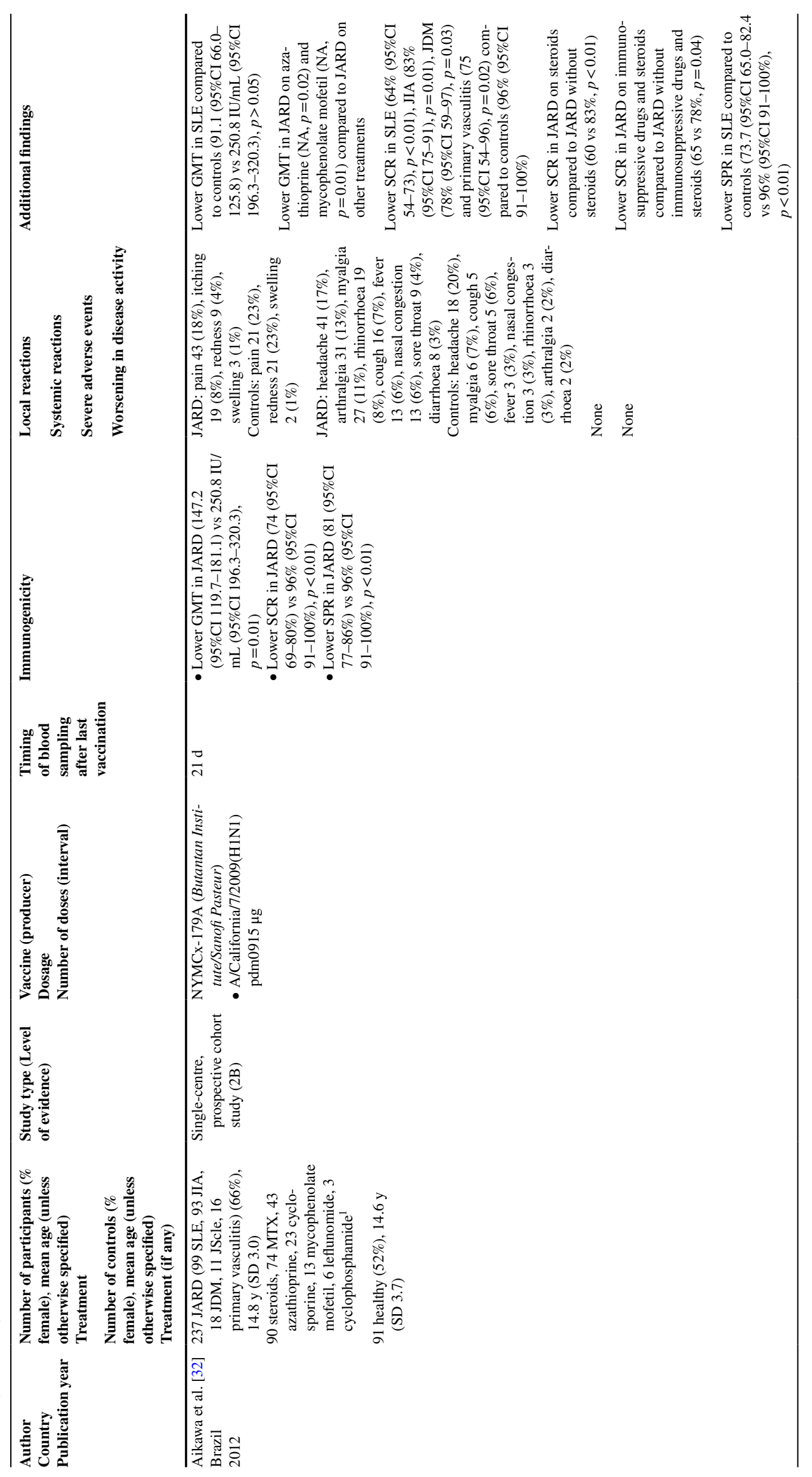




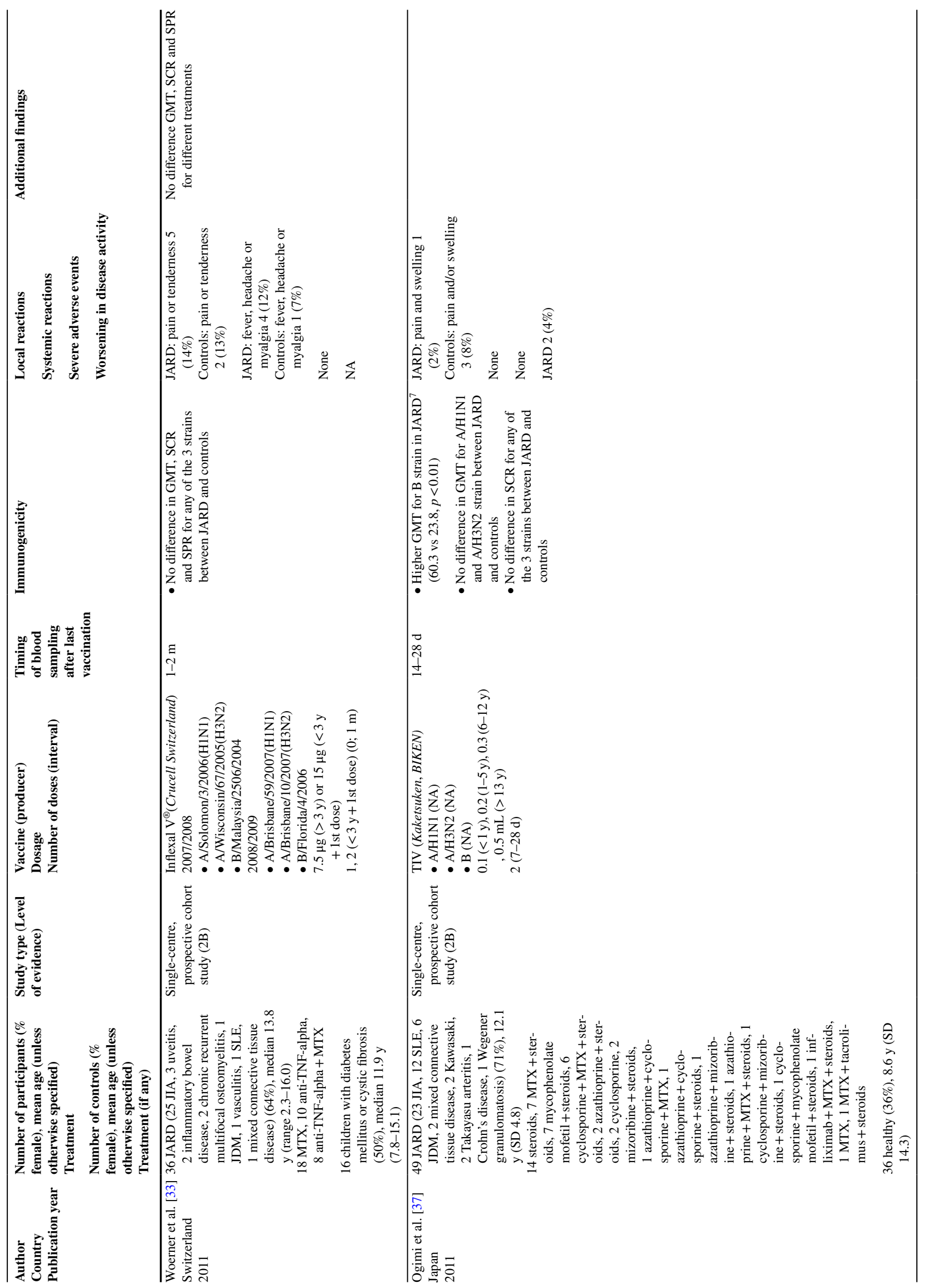




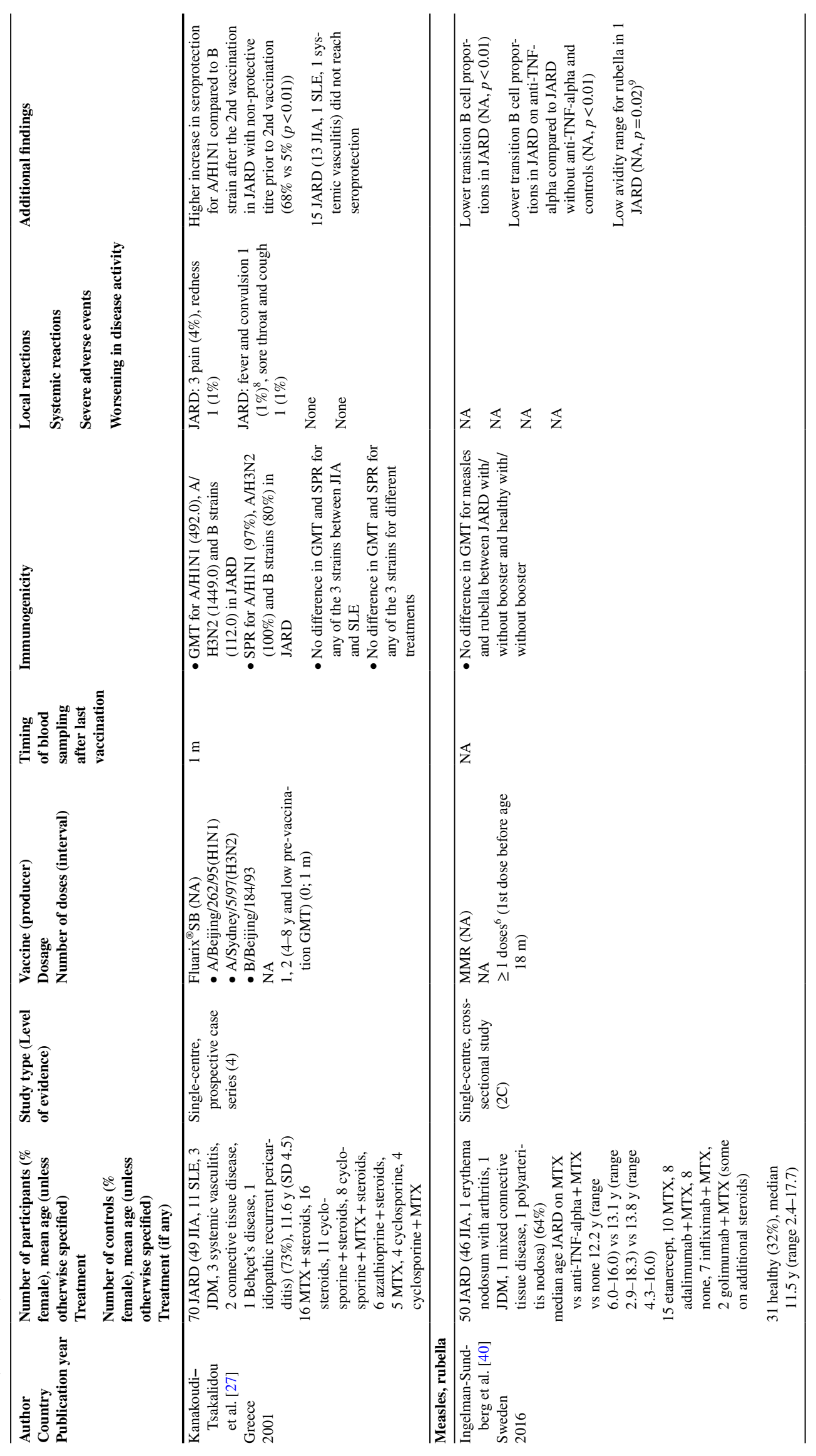




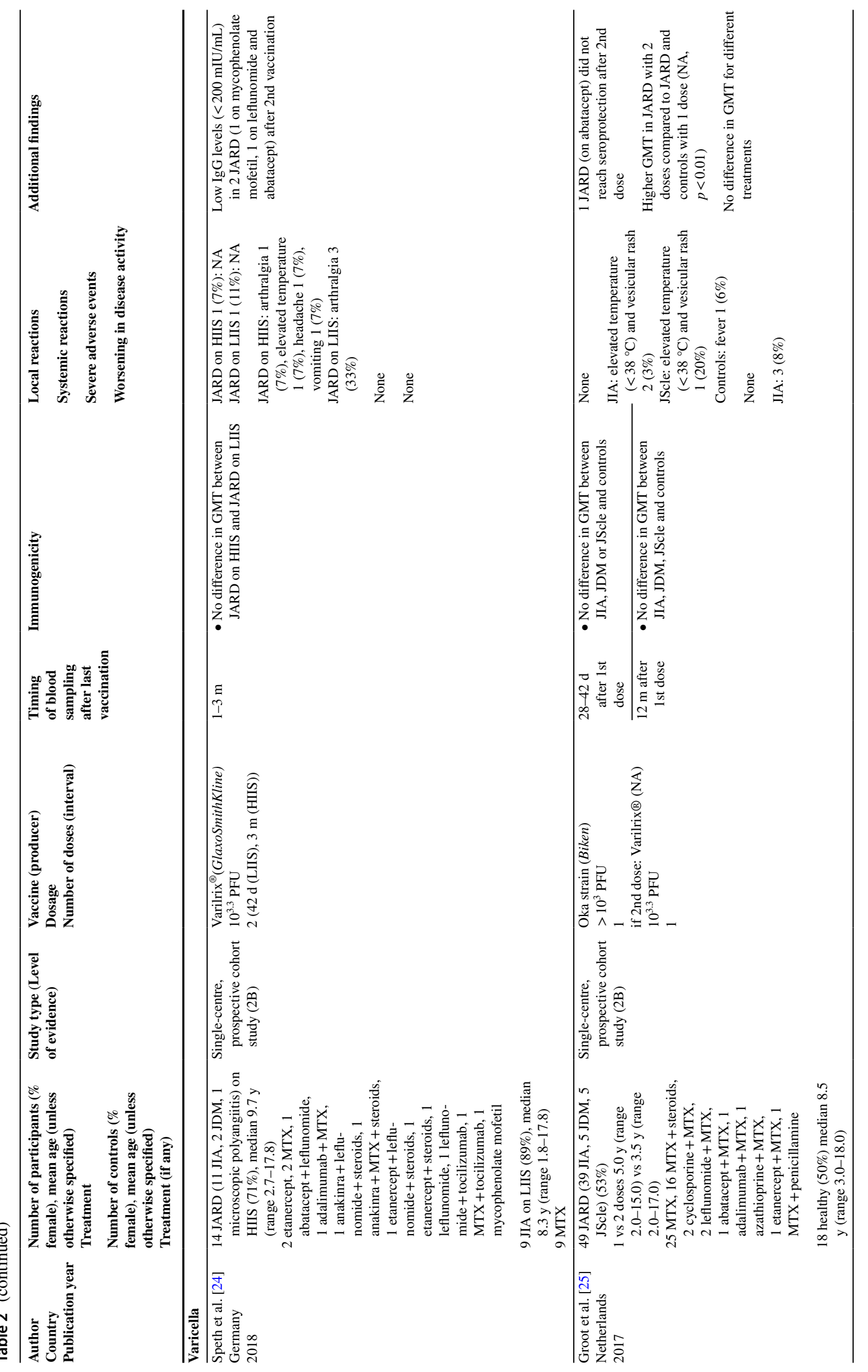

Springer 


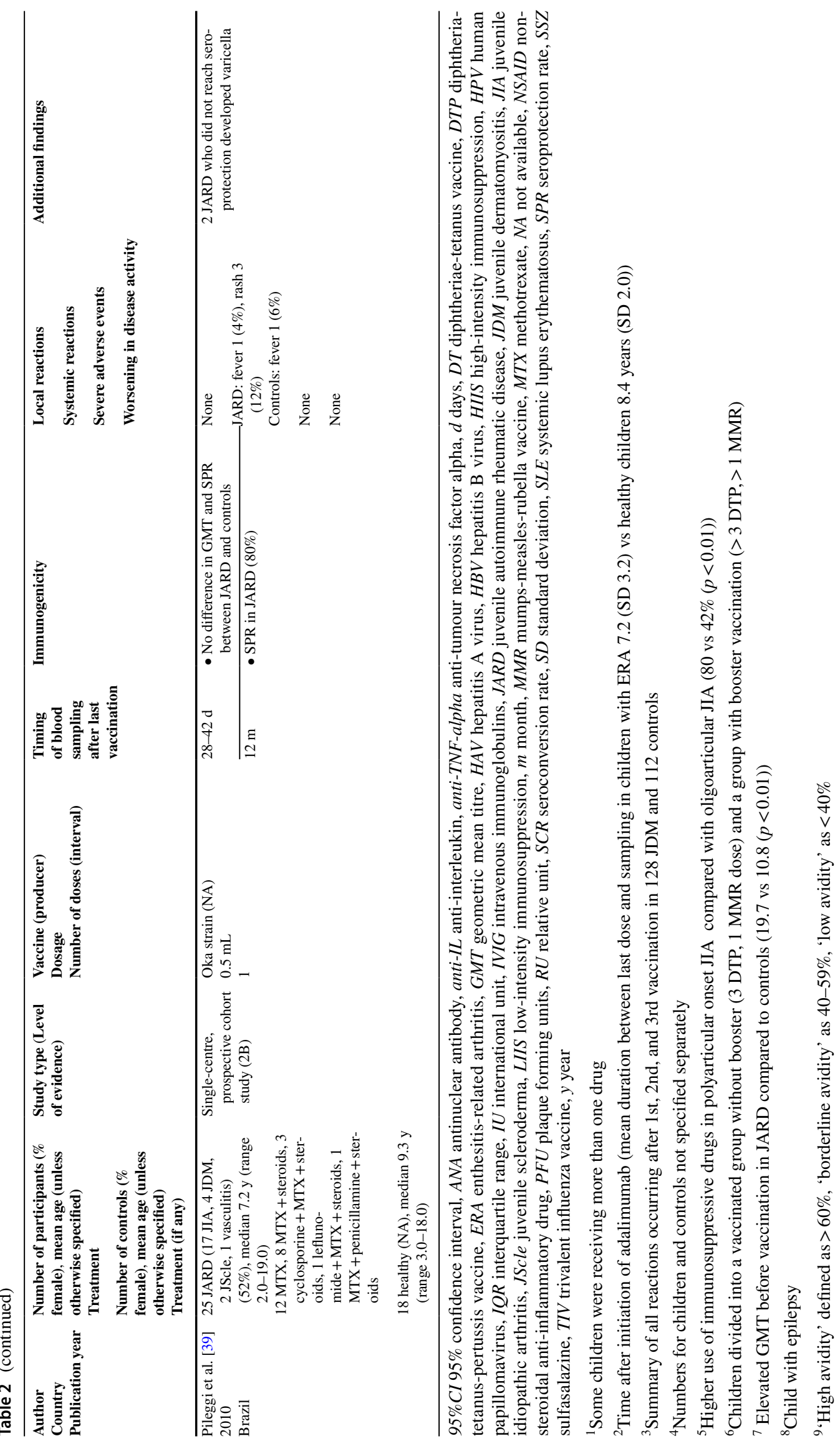


Table 3 Risk of bias summary of studies included in the review

\begin{tabular}{|c|c|c|c|c|c|c|c|c|c|}
\hline Reference & $\begin{array}{l}\text { Publication } \\
\text { year }\end{array}$ & $\begin{array}{l}\text { Study } \\
\text { type }\end{array}$ & Confounding & $\begin{array}{l}\text { Selection } \\
\text { bias }\end{array}$ & $\begin{array}{l}\text { Misclassification } \\
\text { bias }\end{array}$ & $\begin{array}{l}\text { Performance } \\
\text { bias }\end{array}$ & $\begin{array}{l}\text { Attrition } \\
\text { bias }\end{array}$ & $\begin{array}{l}\text { Detection } \\
\text { bias }\end{array}$ & $\begin{array}{l}\text { Reporting } \\
\text { bias }\end{array}$ \\
\hline Maritsi et al. [43] & 2019 & $\mathrm{CS}$ & - & - & - & - & - & - & - \\
\hline Grein et al. [42] & 2020 & $\mathrm{CS}$ & - & + & - & + & + & - & - \\
\hline Guissa et al. [23] & 2012 & $\mathrm{CS}$ & - & - & - & - & - & - & - \\
\hline Maritsi et al. [13] & 2017 & $\mathrm{CS}$ & - & - & - & - & - & - & - \\
\hline Erguven et al. [35] & 2011 & $\mathrm{CS}$ & - & - & - & - & + & - & - \\
\hline Kasapçopur et al. [16] & 2004 & $\mathrm{CS}$ & - & - & - & - & - & - & - \\
\hline Heijstek et al. [44] & 2014 & $\mathrm{CS}$ & - & - & - & + & + & - & - \\
\hline Brunner et al. [41] & 2020 & CSS & - & - & - & - & - & - & - \\
\hline Heijstek et al. [30] & 2012 & CSS & + & + & - & - & - & - & - \\
\hline $\begin{array}{l}\text { Camacho-Lovillo } \\
\text { et al. [12] }\end{array}$ & 2017 & $\mathrm{CS}$ & - & - & - & - & + & - & - \\
\hline Aikawa et al. [22] & 2013 & CS & + & - & - & - & - & - & - \\
\hline Carvalho et al. [46] & 2013 & $\mathrm{CS}$ & - & - & - & + & + & - & - \\
\hline Dell'Era et al. [31] & 2012 & $\mathrm{CS}$ & - & - & - & + & - & - & - \\
\hline Shinoki et al. [29] & 2012 & $\mathrm{CS}$ & + & - & - & - & - & - & - \\
\hline Toplak et al. [28] & 2012 & $\mathrm{CS}$ & + & + & - & - & + & - & - \\
\hline Stoof et al. [20] & 2014 & $\mathrm{CS}$ & + & - & - & - & - & - & - \\
\hline $\begin{array}{l}\text { Zonneveld-Huijssoon } \\
\text { et al. [26] }\end{array}$ & 2007 & $\mathrm{CS}$ & - & - & - & - & - & + & - \\
\hline Aikawa et al. [19] & 2015 & $\mathrm{CS}$ & - & - & - & - & - & - & - \\
\hline Farmaki et al. [38] & 2010 & $\mathrm{CS}$ & + & - & - & - & - & - & - \\
\hline Heijstek et al. [45] & 2013 & RCT & - & - & - & - & + & - & - \\
\hline Borte et al. [14] & 2009 & $\mathrm{CCS}$ & - & + & - & + & - & - & - \\
\hline Aytac et al. [34] & 2011 & $\mathrm{CS}$ & - & + & - & + & - & - & - \\
\hline Miyamoto et al. [36] & 2011 & $\mathrm{CCS}$ & + & - & - & - & - & - & - \\
\hline Kashef et al. [15] & 2008 & $\mathrm{CCS}$ & - & - & - & + & - & - & - \\
\hline Campos et al. [21] & 2013 & $\mathrm{CS}$ & - & + & - & - & - & - & - \\
\hline Barbosa et al. [47] & 2012 & $\mathrm{RCT}$ & - & - & - & - & - & - & - \\
\hline Belderok et al. [17] & 2013 & $\mathrm{CS}$ & + & - & - & - & - & - & - \\
\hline Heijstek et al. [48] & 2013 & $\mathrm{CS}$ & - & - & - & + & - & - & - \\
\hline $\begin{array}{l}\text { Ingelman-Sundberg } \\
\text { et al. [40] }\end{array}$ & 2016 & CSS & + & - & - & + & - & - & - \\
\hline Aikawa et al. [18] & 2013 & $\mathrm{CS}$ & - & - & - & - & + & - & - \\
\hline Aikawa et al. [32] & 2012 & $\mathrm{CS}$ & + & + & - & - & - & - & - \\
\hline Woerner et al. [33] & 2011 & $\mathrm{CS}$ & + & - & - & - & - & - & - \\
\hline Ogimi et al. [37] & 2011 & $\mathrm{CS}$ & + & + & - & - & - & - & - \\
\hline $\begin{array}{l}\text { Kanakoudi-Tsakalidou } \\
\text { et al. [27] }\end{array}$ & 2001 & Cs & - & - & - & - & - & - & - \\
\hline Speth et al. [24] & 2018 & CS & + & - & - & - & + & - & - \\
\hline Groot et al. [25] & 2017 & $\mathrm{CS}$ & + & - & - & - & - & - & - \\
\hline Pileggi et al. [39] & 2010 & $\mathrm{CS}$ & - & - & - & - & - & - & - \\
\hline
\end{tabular}

$C s$ case series, CS cohort study, CCS case-control study, CSS cross-sectional study, $R C T$ randomised controlled trial 


\section{Juvenile dermatomyositis}

Two studies investigated the immunogenicity and safety of vaccines in children with juvenile dermatomyositis (JDM) $[23,42]$. The studies investigated human papilloma virus (HPV) and influenza vaccines in 72 children with JDM and 116 controls.

\section{HPV}

One study investigating the immunogenicity and safety of three doses of a HPV16/HPV18 vaccine in 42 children with JDM and 35 healthy children, 1 and 6 months after vaccination [42]. No difference in SPRs for both serotypes was detected 1 month after the last vaccination. Six months after the last vaccination, SPR for both serotypes was $94 \%$ in children with JDM. SPRs were not specified for healthy children. No difference in SPRs was found between children on different treatment regimens (steroids, hydroxychloroquine, MTX, azathioprine, cyclosporine, or mycophenolate mofetil) at either time point. No SAEs were observed. One child with JDM was reported to have a worsening in disease activity 6 months after vaccination.

\section{Influenza}

One study investigated the immunogenicity and safety of one dose of influenza vaccination (A/H1N1 strain) in 30 children with JDM and 81 healthy children, 21 days after vaccination [23]. A lower SCR was found in children with JDM. In contrast, no difference in GMT or SPR was detected. Separate results for different types of immunosuppressive treatment were not specified. No severe SAEs or worsening in diseases activity were reported.

\section{Juvenile idiopathic arthritis}

A total of 18 studies investigated the immunogenicity and safety of 10 different vaccines (hepatitis A virus (HAV), hepatitis B virus (HBV), HPV, diphtheria, tetanus, influenza, meningococcus C (MenC), measles-mumps-rubella (MMR), pneumococcal polysaccharide, and conjugated vaccine) in 1555 children with JIA on immunosuppressive treatment, 4118 healthy children, and 14 children with non-rheumatic diseases [12-14, 16, 19, 20, 22, 26, 28-31, 35, 38, 41, 4446].

\section{HAV, HBV}

Two studies investigated the immunogenicity and safety of two doses of HAV vaccination in 130 children with JIA and 143 healthy children $[13,35]$. One study found a lower GMT against HAV in children with JIA compared to healthy children 1 and 12 months after vaccination, but no difference in SCR or SPR, while the other study found a lower SPR in children with JIA 2 months after vaccination. The first study reported a worsening in disease activity in $15(18 \%)$ children with JIA (in two after the first dose and in 13 after the second dose after a mean of 8 months) [13]. None of them had a worsening in disease activity during the first 3 months after vaccination. No SAEs were reported in the two studies.

The second study investigated the immunogenicity of three doses of HBV vaccine in 39 children with JIA and 41 healthy children and found a lower GMT and SPR in children with JIA [16]. No difference in GMTs was found between children on steroids and those on MTX. No SAEs were reported.

\section{HPV}

One study investigated the immunogenicity and safety of three doses of a HPV16/18 vaccine in 68 children with JIA and 55 healthy controls [44]. No difference in GMT was found. No difference in GMTs or antibody avidity was found between children on MTX and those on anti-tumournecrosis-factor (TNF)-alpha blockers, anti-interleukin (IL)-1 blockers, leflunomide, and mycophenolate mofetil. One child on MTX did not reach seroprotection. No worsening in disease activitiy was reported. In 14 children with JIA and one healthy child SAEs were reported, many of them were elective hospitalisations or surgeries (for details see Table 2).

\section{Diphtheria, tetanus}

Two studies assessed the immunogenicity of diphtheria and tetanus vaccination in 429 children with JIA and 2176 healthy children [30, 41]. One study found lower GMTs and SPRs for diphtheria and tetanus in children with JIA compared to healthy children [30]. In the other study, no control children were included, the SPR for diphtheria in children with JIA was 90\%, and for tetanus 100\% [41]. No difference in GMTs or SPRs was found between children on abatacept, MTX, steroids, and anti-TNF-alpha blockers [30, 41]. Neither study provided information about SAEs or worsening in disease activity.

\section{Influenza}

Six studies investigated the immunogenicity and safety of one or two doses of influenza vaccines in 292 children with JIA, 154 healthy children, and 14 children with non-rheumatic diseases [12, 22, 28, 29, 31, 46]. Five studies used a trivalent influenza vaccine (TIV) and one study an influenza vaccine with an A/H1N1 strain only. The GMTs were lower against at least one strain in two studies [28, 31], the SCRs were lower against at least one strain in four studies [22, 28, 31, 46], and 
the SPRs were lower for at least one strain in two studies (see Tables 1 and 2) [31, 46]. Most studies did not report differences in specific antibody responses between different treatment regimens [12, 22, 29]. However, one study reported that children on anti-TNF-alpha blockers had lower SCR and SPR against A/H1N1 compared to children on leflunomide, MTX, steroids, and cyclosporine [46]. Only one study specified SAEs; one child with JIA on etanercept needed a hospitalisation for fever and coxalgia 1 day after vaccination [31]. Eighteen children with JIA reported a worsening in disease activity 7 days to 6 months after vaccination [12, 28, 31].

\section{MenC}

Two studies investigated the immunogenicity of one dose of MenC vaccination in 361 children with JIA and 1527 healthy children $[20,26]$. One study found no difference in GMT between children with JIA and healthy ones [20]. The other study, which compared different immunosuppressive treatments in children with JIA, found a lower GMT against MenC in children treated with MTX, sulfasalazine, etanercept, infliximab, or cyclosporine compared to children on non-steroidal anti-inflammatory drugs or without treatment [26]. No information concerning SAEs was provided. No worsening in disease activity was reported.

\section{Pneumococcus}

One study each investigated the immunogenicity and safety of one dose of 23-valent pneumococcal polysaccharide vaccine (PPV23) and two doses of 7-valent pneumococcal conjugate vaccine (PCV7) in 27 and 63 children with JIA, respectively [19, 38]. Both studies compared the antibody response to pneumococcus in children with JIA on MTX and cyclosporine with children with JIA on the former treatment plus additional adalimumab or etanercept. The study using the PPV23 vaccine found no difference in GMTs, SCRs, or SPRs for serotypes 4, 6B, $9 \mathrm{~V}, 14,18 \mathrm{C}, 19 \mathrm{~F}$, and 23F between the two groups [19]. The study using the PCV7 vaccine found lower GMTs but not SPRs for serotypes 4, 14, and $23 \mathrm{~F}$ in on adalimumab or etanercept compared to those on steroids or cyclosporine [38]. However, no difference in GMTs was found between children on adalimumab and those on etanercept [38]. One child on etanercept developed pneumococcal pneumonia (serotype not specified) requiring hospitalisation 5 months after vaccination [19]. In one child with JIA on additional adalimumab or etanercept, a worsening in disease activity was reported [38].

\section{MMR}

Three studies, including one RCT, investigated the immunogenicity of MMR vaccination in 546 children with JIA and
2198 healthy children $[14,30,45]$. In the RCT, both groups included children with JIA on immunosuppressive treatment who had been previously been vaccinated with MMR, but only one group was randomised to receive a MMR booster [45]. In the other two studies, either two doses of MMR or a monovalent measles vaccine followed by MMR were given [14, 30].

One study reported a higher GMT against measles in children with JIA compared to healthy children [30]. However, the children with JIA were older than the healthy children; therefore, likely more of them had received an MMR booster, which was given at nine years of age. Another study reported a lower GMT against measles in children with JIA after receiving MTX for 6 months initiated more than 4 years after the second dose of MMR compared to healthy children [14]. But again the children were from different age groups. The children with JIA had a mean age of 16 years compared to the healthy children with a mean age of 11 years. Therefore, there was a larger time interval between vaccination and measuring vaccine antibody responses in children with JIA. Only one study measured SPRs for measles and reported a higher SPR in children with JIA compared to healthy children in the group vaccinated with two doses of MMR but not in the group vaccinated with a monovalent measles vaccine followed by a dose of MMR [30]. However, as mentioned above some of the healthy children, who were younger, likely did not yet receive two doses of the MMR vaccine. The RCT reported an increase in GMT in the children with JIA who received an MMR booster 12 months after the vaccine [45].

Two studies compared GMTs against mumps in children with JIA compared to healthy children [14, 30]. One found a lower GMT against mumps in children with JIA, while the other did not find a difference in GMTs. The one study which measured SPRs for mumps reported no difference in children with JIA compared to the healthy controls when receiving two doses of MMR but a lower SPR for mumps in the children who received a monovalent measles vaccine followed by one dose of MMR [30]. The RCT reported an increase in GMT against mumps in the children with JIA who received an MMR booster 12 months after vaccination [45].

One study did not detect a difference in rubella specific GMTs between children with JIA and healthy children, while another study found a lower GMT in children with JIA $[14,30]$. The study which measured SPRs against rubella, reported a lower SPR in children with JIA compared to healthy children after one dose of monovalent measles vaccine followed by MMR but not in the children who received two doses of MMR [30]. The RCT reported a higher GMT in children with JIA receiving a MMR booster compared to children with JIA without the booster 12 months after vaccination [45]. 
No difference in GMTs against measles, mumps, or rubella was found between children on MTX and children on steroids, anti-TNF-alpha blockers, anti-IL-1 blockers, or leflunomide [30, 45].

Only two of the three studies evaluated the safety of the MMR vaccine; no worsening in disease activity was reported in either of the studies $[14,45]$. Only the RCT reported SAEs; five children who received a booster and eleven children in the control group were reported to have SAEs [45]. Most of them were elective hospitalisation and surgeries, unlikely related to the vaccination. No disease due to infections with vaccine viruses was observed [45].

\section{Systemic lupus erythematosus}

Five studies including 236 children with systemic lupus erythematosus (SLE) and 228 healthy children investigated the immunogenicity and safety of five different vaccines (HBV, tetanus, influenza, measles, and varicella (VZV)) $[15,21,34,36,47]$.

\section{HBV}

One study investigated the immunogenicity and safety after three doses of an HBV vaccine in 20 children with SLE and 24 healthy children [34]. A lower GMT was found in children with SLE, while the SPR was similar in both groups. Separate results for different types of immunosuppressive treatment were not reported. No SAEs were reported. Three children with SLE were reported to have a worsening in disease activity, one child 1 month after the first dose and the other two children 1 month after the second dose.

\section{Tetanus}

Two studies investigated the specific antibody response after five doses of tetanus vaccine in 70 children with SLE and 74 healthy children $[15,36]$. In one study, a lower GMT was found in children with inactive SLE (but not active SLE) compared to healthy children [36]. The other study did not find a difference in GMTs between children with SLE and healthy ones [15]. Separate results for different types of immunosuppressive treatment were not reported. During the study, four SAEs occurred in children with SLE; all were disseminated infections requiring hospital admission unlikely related to the vaccine (disseminated varicella infection, primary peritonitis, meningitis and pneumonia) [15]. No information concerning worsening in disease activity was provided in either study $[15,36]$.

\section{Influenza}

One study investigates the immunogenicity of one dose of an A/H1N1 influenza vaccine in 118 children with SLE and
102 healthy children [21]. A lower GMT, SCR, and SPR were found in children with SLE. Children on a higher steroid dose did more frequently not seroconvert compared to those on a lower dose (18.0 vs $10.5 \mathrm{mg}$ per day). No SAEs or worsening in disease activity were reported.

\section{Measles}

One retrospective study investigated the specific antibody response after measles vaccination in 30 children with SLE with different immunosuppressive treatments (chloroquine, steroids, azathioprine, cyclosporine, cyclophosphamide, and MTX) and 28 healthy children [36]. No difference in GMTs was found between children with active SLE, inactive SLE and healthy children. Separate results for different types of immunosuppressive treatment were not reported. No information concerning SAEs and worsening in disease activity was provided.

\section{VZV}

One RCT investigated the immunogenicity and safety of one dose of VZV vaccine in 28 children with SLE on either chloroquine, steroids, azathioprine, or MTX and 28 healthy children [47]. No difference in GMT for children with JIA compared to healthy ones was found 1,6 , and 12 months after vaccination. Separate results for different types of immunosuppressive treatment were not reported. Furthermore, no SAEs or worsening in disease activity were reported.

\section{Other JARDs}

Eleven studies including 667 children with different JARDs, 254 healthy children, and 16 children with non-rheumatic diseases investigated the immunogenicity and safety of HAV, HBV, HPV, tetanus, influenza, measles, rubella, and VZV vaccines $[17,18,24,25,27,32,33,37,39,40,48]$.

\section{HAV, HBV}

One study investigated the immunogenicity of two doses of a combined HAV and HBV vaccines in 78 children with JARD, including 71 (91\%) children with JIA [17]. A positive SCR was reached for HAV and HBV in $100 \%$ and $93 \%$, respectively. No difference in GMTs for different treatments was found. No information concerning SAEs and worsening in disease activity was provided.

\section{HPV}

One study investigated the immunogenicity of three doses of a HPV in 12 children with JARD (6 JDM, 6 SLE) and 49 healthy children [48]. No difference in GMTs between 
JARD and controls were found 1 and 6 months after vaccination. One child with JDM without immunosuppressive treatment did not seroconvert. Separate results different types of immunosuppressive treatment were not reported. No information concerning SAEs was provided. One child with JDM was reported to have a worsening in disease activity 1 month after the second vaccination.

\section{Tetanus}

One study investigated the immunogenicity of more than three doses of a tetanus vaccine in 50 children with JARD (including 46 (92\%) children with JIA) and 31 healthy children [40]. A lower GMT was detected in children with JARD on MTX with or without anti-TNF-alpha blockers compared to children with JARD without treatment or healthy children. No information concerning SAEs and worsening in disease activity was provided.

\section{Influenza}

Five studies investigated the immunogenicity of influenza vaccination in 430 children with JARD, 138 healthy children, and 16 children with non-rheumatic diseases $[18,27,32,33,37]$. Three studies used a TIV and two studies a single A/H1N1 strain vaccine. Two studies did not find differences in GMT, SCR, or SPR between children with JARD and controls $[18,33]$. One study found a lower GMT, SCR, and SPR for the A/H1N1 strain in children with JARD compared to healthy children [32]. In contrast in another study, a higher GMT for the B strain in children with JARD compared to healthy children was found, while there was no difference in SCR for all three strains [37]. One study reported a lower GMT in children on azathioprine, mycophenolate mofetil, and steroids compared to children on cyclosporine, leflunomide, or cyclophosphamide [32]. No SAEs were reported [18, 27, 32, 33, 37]. In one study a worsening in disease activity was reported in two children with JARD (one child with JIA, one with Takayasu arteritis) 2 weeks after vaccination [37].

\section{Measles, rubella}

One study investigated the specific antibody responses against measles and rubella after a minimum of one dose of MMR in 50 children with JARD (46 children with JIA) on MTX or MTX plus anti-TNF-alpha blockers and 31 healthy children [40]. No difference in GMTs for measles or rubella was found between children with JARD and healthy children. Children with JARD on anti-TNF-alpha blockers had a lower proportion of transition B cells compared with those without antiTNF-alpha blockers and controls. No information concerning SAEs and worsening in disease activity was provided.

\section{VZV}

Three studies investigated the immunogenicity of one or two doses of VZV vaccination in 97 children with JARD on different immunosuppressive treatments and 36 healthy children $[24,25,39]$. No difference in GMT or SPR was found in any of the three studies. No SAEs were reported. Separate results for different types of immunosuppressive treatment were not reported. One study reported a worsening in disease activity in three children with JIA 4 to 6 weeks after vaccination [25].

\section{Discussion}

Our systematic review shows that vaccines in children and adolescents with JARDs on immunosuppressive treatment are safe and immunogenic. Overall, a decreased specific antibody response was reported in one-third (26-33\%) of all measurements (GMT, SPR, or SCR). However, it is important to take into consideration the timing of the measurements. For example, a study, which measured antibodies to $\mathrm{HAV} / \mathrm{HBV}$ vaccine in children with JARD, reported that the initial response after one dose was low, but after receiving a second dose, almost all children with JARD seroconverted [17]. This stresses the importance for the completion of vaccination schedules, especially in high-risk children, such as children with JARDs.

Furthermore, due to the lower vaccine response found in approximately one-third of children with JARDs on immunosuppressive treatment, additional booster doses can be offered to optimise vaccine efficacy in these children. Two studies showed an accelerated antibody loss in children with JIA [6, 26]. In the included RCT, which randomised children with JIA to either receive or not receive a booster dose of MMR, a higher proportion of children who did not receive a booster were not seroprotected [45]. This demonstrates the importance of booster doses in children with JARDs. A pro-active approach to detect insufficient antibodies levels in children with JARDs might be a valuable tool to optimise the timing booster doses. Similarly, pre-travelling antibody measurements could be useful, for example, for HAV in children who are travelling to high incidence countries.

A further main concern in children with inflammatory or autoimmune diseases, including JARD, is that vaccines may trigger an onset or worsening in disease activity. For example, it has previously been reported that HBV vaccination might trigger the onset of an underlying inflammatory or autoimmune rheumatic disease [49] or that HPV vaccination could trigger the onset of SLE [50]. Many studies exclude children with active JARD to avoid potential worsening in disease activity [34]. Only one study included in the review, compared the immunogenicity of a measles and tetanus 
vaccine in children with active and inactive SLE [36]. The study reported a higher immunogenicity of the vaccines in children with active compared to inactive SLE. However, the children in the latter group were older than the ones with active SLE, which likely explains some of this difference.

In our review, ten studies reported children with worsening in disease activity after vaccination $[12,13,25,28,31$, $34,37,38,42,48]$. However, often it could not be differentiated between the vaccines as a trigger for the worsening of disease or other possibilities causes, such as changes or non-compliance to the immunosuppressive treatment [31]. Furthermore, many of the JARDs are characterised by an intermittent and relapsing course even without triggers. As many care providers prefer to vaccinate these children in a stable phase and often defer vaccination until such a phase is reached, the chance of a relapse due to the normal course of the disease might be higher after vaccination, which can then be misinterpreted as a relapse triggered by the vaccine. Although, worsening in disease activity might be a more of a concern after live attenuated vaccines, only one study reported a worsening in disease activity in three of 39 children with JIA, 4 to 6 weeks after VZV vaccination [25]. In contrast, one of the studies reported a decrease in number of affected joints in children with JARD after VZV vaccination [39]. Another study included in the review, investigated the association of autoantibodies and disease activity after influenza vaccination in children with JIA, and did not find an association [28]. Importantly, it should be noted and communicated that vaccine-preventable diseases, for example mumps, measles, and rubella, can also trigger an activation or exacerbation of the underlying inflammatory or autoimmune JARD [51, 52]. Overall, there is increasing evidence suggesting that vaccines do not induce significant worsening of underlying disease $[8,53]$.

Not only for a worsening in disease activity, but also for SAEs, it is difficult to proof a correlation. Many of the SAEs reported in the studies included in this review seem unlikely to be correlated to vaccination (e.g. elective hospitalisations and surgeries). As an example, two children with SLE have been reported to have deterioration in renal function within 18 months after HPV vaccinations [54]. However, it is not clear if this is an adverse event due to the vaccination or rather illustrate the natural progression of the disease itself. As both cases were diagnosed with state four nephritis before vaccination, an evolution of the disease itself seems more likely. In another study, a febrile convulsion was reported as a severe adverse event after influenza vaccination in a child with JARD. However, this child was known for epilepsy [27]. In one study, a vesicular rash was reported in three of 49 children with JARD on immunosuppressive treatment with MTX and anti-TNF-alpha blockers [25]. Another study reported that rashes after VZV vaccination were not more common in children with SLE on immunosuppressive treatment compared to healthy children [47]. None of the studies reported an infection due to attenuated vaccine viruses, and all recovered promptly without treatment. These results are, however, limited by most studies investigating live attenuated vaccine not reporting systemic reactions, severe adverse events, or worsening in disease activity [14, 30, 36, 40, 43].

Unfortunately, there is no study which investigated the immunogenicity and safety of yellow fever vaccination in children with JARDs on immunosuppressive treatment. A recent review from the European League Against Rheumatism concluded that yellow fever vaccination in adults with autoinflammatory rheumatic diseases should be avoided due to the risk of vaccine-induced yellow fever [55]. As yellow fever is circulating in many parts of the world, the safety and immunogenicity of the vaccine in children with JARDs is an urgent future research topic.

Furthermore, the current severe acute respiratory syndrome coronavirus type 2 (SARS-CoV-2) pandemic also highlights the urgent need to assess the immunogenicity and safety of new vaccines, such as messenger ribonucleic acid-based (mRNA) vaccines, in this patient group. Three recent studies showed that mRNA and viral vector-based SARS-CoV-2 vaccines are immunogenic and safe (no increased side effects or induction of disease flares) in adults with rheumatic diseases on immunosuppressive treatment [56-58]. However, specific antibody responses were lower in adults on immunosuppressive treatment compared to healthy controls, especially those on steroids, rituximab, mycophenolate mofetil, and abatacept $[56,58]$. No data is currently available on the immunogenicity and safety of SARS-CoV-2 vaccine in children with rheumatic diseases on immunosuppressive treatment.

The strengths of our review are the systematic approach and the comprehensive literature search. The limitations are the heterogenicity of the included studies which precluded a meta-analysis, especially as vaccine responses were not always measured one month after vaccination (gold-standard) and sometimes the time interval between vaccination and measurement of responses even differed between cases and controls. Furthermore, the dose of the immunosuppressive treatment and the disease activity or severity was not specified in most of the studies. Many of the studies were underpowered, and not designed to show non-inferiority between children with JARD and controls; therefore, finding no difference between the groups does not imply equivalence. Moreover, we only evaluated antibody responses, as there are almost no studies which report on vaccine efficacy, and cellular or cytokine responses to vaccines.

In conclusion, vaccination in children with JARD on immunosuppressive treatment should be promoted and the importance for the completion of vaccination schedules 
should be stressed. Strategies to compensate for the lower vaccine responses or faster decline of antibodies include measuring antibody levels to determine the optimal timing for the administration of additional booster doses. Further studies including children with active JARD are needed for evidence-based guidelines to vaccination in these children.

Supplementary Information The online version contains supplementary material available at https://doi.org/10.1007/s00431-021-04283-w.

Authors' contributions MK and PZ designed the study. MK did the literature search and drafted the initial manuscript. LFP and PZ critically revised the manuscript, and all authors approved the final manuscript as submitted.

\section{Availability of data and material N/A}

Code availability N/A

\section{Declarations}

\section{Ethics approval N/A}

Consent to participate N/A

\section{Consent for publication N/A}

Conflict of interest The authors declare no competing interests.

Open Access This article is licensed under a Creative Commons Attribution 4.0 International License, which permits use, sharing, adaptation, distribution and reproduction in any medium or format, as long as you give appropriate credit to the original author(s) and the source, provide a link to the Creative Commons licence, and indicate if changes were made. The images or other third party material in this article are included in the article's Creative Commons licence, unless indicated otherwise in a credit line to the material. If material is not included in the article's Creative Commons licence and your intended use is not permitted by statutory regulation or exceeds the permitted use, you will need to obtain permission directly from the copyright holder. To view a copy of this licence, visit http://creativecommons.org/licenses/by/4.0/.

\section{References}

1. Stanton BF (2018) Pediatric rheumatology: a field of great progress. Pediatr Clin North Ame. 65(4):xiii-xiv

2. Marzetti V, Breda L, Miulli E et al (2017) Clinical characteristics of juvenile idiopathic arthritis in an area of central Italy: a population-based study. Ann Ig 29(4):281-292

3. Ada G (2005) Overview of vaccines and vaccination. Mol Biotechnol 29(3):255-272

4. Bizjak M, Blazina Š, Zajc Avramovič M, Markelj G, Avčin T, Toplak N (2020) Vaccination coverage in children with rheumatic diseases. Clin Exp Rheumatol 38(1):164-170

5. Morin MP, Quach C, Fortin E, Chédeville G (2012) Vaccination coverage in children with juvenile idiopathic arthritis followed at a paediatric tertiary care centre. Rheumatology (Oxford) 51(11):2046-2050
6. Maritsi D, Vartzelis G, Soldatou A, Garoufi A, Spyridis N (2013) Markedly decreased antibody titers against hepatitis B in previously immunised children presenting with juvenile idiopathic arthritis. Clin Exp Rheumatol 31(6):969-973

7. Jaeger VK, Hoffman HM, van der Poll T et al (2017) Safety of vaccinations in patients with cryopyrin-associated periodic syndromes: a prospective registry based study. Rheumatology (Oxford) 56(9):1484-1491

8. Heijstek MW, Ott de Bruin LM, Bijl M et al (2011) EULAR recommendations for vaccination in paediatric patients with rheumatic diseases. Ann Rheum Dis 70(10):1704-1712

9. Groot N, Heijstek MW, Wulffraat NM (2015) Vaccinations in paediatric rheumatology: an update on current developments. Curr Rheumatol Rep 17(7):46

10. Moher D, Liberati A, Tetzlaff J, Altman DG (2009) Preferred reporting items for systematic reviews and meta-analyses: the PRISMA statement. Bmj 339:b2535

11. Sterne JA, Hernan MA, Reeves BC et al (2016) ROBINS-I: a tool for assessing risk of bias in non-randomised studies of interventions. BMJ 355:i4919

12. Camacho-Lovillo MS, Bulnes-Ramos A, Goycochea-Valdivia W et al (2017) Immunogenicity and safety of influenza vaccination in patients with juvenile idiopathic arthritis on biological therapy using the microneutralization assay. Pediatr Rheumatol Online J 15(1):62

13. Maritsi DN, Coffin SE, Argyri I, Vartzelis G, Spyridis N, Tsolia MN (2017) Immunogenicity and safety of the inactivated hepatitis A vaccine in children with juvenile idiopathic arthritis on methotrexate treatment: a matched case-control study. Clin Exp Rheumatol 35(4):711-715

14. Borte S, Liebert UG, Borte M, Sack U (2009) Efficacy of measles, mumps and rubella revaccination in children with juvenile idiopathic arthritis treated with methotrexate and etanercept. Rheumatology 48(2): 144-148

15. Kashef S, Ghazizadeh F, Derakhshan A, Farjadian S, Alyasin S (2008) Antigen-specific antibody response in juvenile-onset SLE patients following routine immunization with tetanus toxoid. Iran J Immunol 5(3):181-184

16. Kasapcopur O, Cullu F, Kamburoglu-Goksel A et al (2004) Hepatitis B vaccination in children with juvenile idiopathic arthritis. Ann Rheum Dis 63(9):1128-1130

17. Belderok SM, Sonder GJ, van Rossum M et al (2013) Evaluation of immune responses to combined hepatitis $\mathrm{A}$ and $\mathrm{B}$ vaccine in HIV-infected children and children on immunosuppressive medication. Vaccine 31(38):4156-4163

18. Aikawa NE, Trudes G, Campos LM et al (2013) Immunogenicity and safety of two doses of a non-adjuvanted influenza A H1N1/2009 vaccine in young autoimmune rheumatic diseases patients. Lupus 22(13):1394-1398

19. Aikawa NE, Franca IL, Ribeiro AC, Sallum AM, Bonfa E, Silva CA (2015) Short and long-term immunogenicity and safety following the 23-valent polysaccharide pneumococcal vaccine in juvenile idiopathic arthritis patients under conventional DMARDs with or without anti-TNF therapy. Vaccine 33(5):604-609

20. Stoof SP, Heijstek MW, Sijssens KM et al (2014) Kinetics of the long-term antibody response after meningococcal $\mathrm{C}$ vaccination in patients with juvenile idiopathic arthritis: a retrospective cohort study. Ann Rheum Dis 73(4):728-734

21. Campos LM, Silva CA, Aikawa NE et al (2013) High disease activity: an independent factor for reduced immunogenicity of the pandemic influenza a vaccine in patients with juvenile systemic lupus erythematosus. Arthritis Care Res 65(7):1121-1127

22. Aikawa NE, Campos LM, Goldenstein-Schainberg C et al (2013) Effective seroconversion and safety following the pandemic influenza vaccination (anti-H1N1) in patients with juvenile idiopathic arthritis. Scand J Rheumatol 42(1):34-40 
23. Guissa VR, Pereira RM, Sallum AM et al (2012) Influenza A H1N1/2009 vaccine in juvenile dermatomyositis: reduced immunogenicity in patients under immunosuppressive therapy. Clin Exp Rheumatol 30(4):583-588

24. Speth F, Hinze CH, Andel S, Mertens T, Haas JP (2018) Varicella-zoster-virus vaccination in immunosuppressed children with rheumatic diseases using a pre-vaccination check list. Pediatr Rheumatol Online J 16(1):15

25. Groot N, Pileggi G, Sandoval CB et al (2017) Varicella vaccination elicits a humoral and cellular response in children with rheumatic diseases using immune suppressive treatment. Vaccine 35(21):2818-2822

26. Zonneveld-Huijssoon E, Ronaghy A, Van Rossum MA et al (2007) Safety and efficacy of meningococcal c vaccination in juvenile idiopathic arthritis. Arthritis Rheum 56(2):639-646

27. Kanakoudi-Tsakalidou F, Trachana M, Pratsidou-Gertsi P, Tsitsami E, Kyriazopoulou-Dalaina V (2001) Influenza vaccination in children with chronic rheumatic diseases and long-term immunosuppressive therapy. Clin Exp Rheumatol 19(5):589-594

28. Toplak N, Subelj V, Kveder T et al (2012) Safety and efficacy of influenza vaccination in a prospective longitudinal study of 31 children with juvenile idiopathic arthritis. Clin Exp Rheumatol 30(3):436-444

29. Shinoki T, Hara R, Kaneko U et al (2012) Safety and response to influenza vaccine in patients with systemic-onset juvenile idiopathic arthritis receiving tocilizumab. Mod Rheumatol 22(6):871-876

30. Heijstek MW, van Gageldonk PG, Berbers GA, Wulffraat NM (2012) Differences in persistence of measles, mumps, rubella, diphtheria and tetanus antibodies between children with rheumatic disease and healthy controls: a retrospective cross-sectional study. Ann Rheum Dis 71(6):948-954

31. Dell'Era L, Corona F, Daleno C, Scala A, Principi N, Esposito S (2012) Immunogenicity, safety and tolerability of MF59adjuvanted seasonal influenza vaccine in children with juvenile idiopathic arthritis. Vaccine 30(5):936-940

32. Aikawa NE, Campos LM, Silva CA et al (2012) Glucocorticoid: major factor for reduced immunogenicity of 2009 influenza A (H1N1) vaccine in patients with juvenile autoimmune rheumatic disease. J Rheumatol 39(1):167-173

33. Woerner A, Sauvain MJ, Aebi C, Otth M, Bolt IB (2011) Immune response to influenza vaccination in children treated with methotrexate or/and tumor necrosis factor-alpha inhibitors. Hum Vaccin 7(12):1293-1298

34. Aytac MB, Kasapcopur O, Aslan M, Erener-Ercan T, Cullu-Cokugras F, Arisoy N (2011) Hepatitis B vaccination in juvenile systemic lupus erythematosus. Clin Exp Rheumatol 29(5):882-886

35. Erguven M, Kaya B, Hamzah OY, Tufan F (2011) Evaluation of immune response to hepatitis $A$ vaccination and vaccine safety in juvenile idiopathic arthritis. J Chin Med Assoc 74(5):205-208

36. Miyamoto M, Ono E, Barbosa C et al (2011) Vaccine antibodies and $\mathrm{T}$ - and B-cell interaction in juvenile systemic lupus erythematosus. Lupus 20(7):736-744

37. Ogimi C, Tanaka R, Saitoh A, Oh-Ishi T (2011) Immunogenicity of influenza vaccine in children with pediatric rheumatic diseases receiving immunosuppressive agents. Pediatr Infect Dis J 30(3):208-211

38. Farmaki E, Kanakoudi-Tsakalidou F, Spoulou V et al (2010) The effect of anti-TNF treatment on the immunogenicity and safety of the 7-valent conjugate pneumococcal vaccine in children with juvenile idiopathic arthritis. Vaccine 28(31):5109-5113

39. Pileggi GS, de Souza CB, Ferriani VP (2010) Safety and immunogenicity of varicella vaccine in patients with juvenile rheumatic diseases receiving methotrexate and corticosteroids. Arthritis Care Res 62(7):1034-1039

40. Ingelman-Sundberg HM, Laestadius A, Chrapkowska C et al (2016) Diverse effects on vaccine-specific serum IgG titres and memory B cells upon methotrexate and anti-TNF-alpha therapy in children with rheumatic diseases: A cross-sectional study. Vaccine 34(10):1304-1311

41. Brunner HI, Tzaribachev N, Cornejo GV et al (2020) Maintenance of antibody response to diphtheria/tetanus vaccine in patients aged 2-5 years with polyarticular-course juvenile idiopathic arthritis receiving subcutaneous abatacept. Pediatr Rheumatol Online J18(1):19

42. Grein IHR, Pinto NBF, Groot N et al (2020) Safety and immunogenicity of the quadrivalent human papillomavirus vaccine in patients with juvenile dermatomyositis: a real-world multicentre study. Pediatr Rheumatol Online J 18(1):87

43. Maritsi DN, Kopsidas I, Vartzelis G, Spyridis N, Tsolia MN (2019) Long-term preservation of measles and rubella specific-IgG antibodies in children with enthesitis related arthritis on anti-TNFo treatment: a prospective controlled study. Rheumatology (Oxford) 58(9):1686-1688

44. Heijstek MW, Scherpenisse M, Groot $\mathrm{N}$ et al (2014) Immunogenicity and safety of the bivalent HPV vaccine in female patients with juvenile idiopathic arthritis: a prospective controlled observational cohort study. Ann Rheum Dis 73(8):1500-1507

45. Heijstek MW, Kamphuis S, Armbrust W et al (2013) Effects of the live attenuated measles-mumps-rubella booster vaccination on disease activity in patients with juvenile idiopathic arthritis: a randomized trial. JAMA 309(23):2449-2456

46. Carvalho LM, de Paula FE, Silvestre RV et al (2013) Prospective surveillance study of acute respiratory infections, influenza-like illness and seasonal influenza vaccine in a cohort of juvenile idiopathic arthritis patients. Pediatr Rheumatol Online J 11:10

47. Barbosa CM, Terreri MT, Rosário PO, de Moraes-Pinto MI, Silva CA, Hilário MO (2012) Immune response and tolerability of varicella vaccine in children and adolescents with systemic lupus erythematosus previously exposed to varicella-zoster virus. Clin Exp Rheumatol 30(5):791-798

48. Heijstek MW, Scherpenisse M, Groot N, Wulffraat NM, Van Der Klis FR (2013) Immunogenicity of the bivalent human papillomavirus vaccine in adolescents with juvenile systemic lupus erythematosus or juvenile dermatomyositis. J Rheumatol 40(9):1626-1627

49. Maillefert JF, Sibilia J, Toussirot E et al (1999) Rheumatic disorders developed after hepatitis B vaccination. Rheumatology (Oxford) 38(10):978-983

50. Gatto M, Agmon-Levin N, Soriano A et al (2013) Human papillomavirus vaccine and systemic lupus erythematosus. Clin Rheumatol 32(9):1301-1307

51. Minden K, Niewerth M, Borte M, Singendonk W, Haas JP (2007) Immunization in children and adolescents with rheumatic diseases. Z Rheumatol 66(2):111-112, 114-118, 120

52. Tingle AJ, Allen M, Petty RE, Kettyls GD, Chantler JK (1986) Rubella-associated arthritis. I. Comparative study of joint manifestations associated with natural rubella infection and RA 27/3 rubella immunisation. Ann Rheum Dis 45(2):110-114

53. Papp KA, Haraoui B, Kumar D et al (2019) Vaccination guidelines for patients with immune-mediated disorders on immunosuppressive therapies. J Cutan Med Surg 23(1):50-74

54. Soybilgic A, Onel KB, Utset T, Alexander K, Wagner-Weiner L (2013) Safety and immunogenicity of the quadrivalent HPV vaccine in female Systemic Lupus Erythematosus patients aged 12 to 26 years. Pediatr Rheumatol Online J 11:29

55. Furer V, Rondaan C, Heijstek MW et al (2020) 2019 update of EULAR recommendations for vaccination in adult patients with autoimmune inflammatory rheumatic diseases. Ann Rheum Dis 79(1):39-52

56. Geisen UM, Berner DK, Tran F et al (2021) Immunogenicity and safety of anti-SARS-CoV-2 mRNA vaccines in patients with chronic inflammatory conditions and immunosuppressive therapy in a monocentric cohort. Ann Rheum Dis 80(10):1306-1311 
57. Sattui SE, Liew JW, Kennedy K et al (2021) Early experience of COVID-19 vaccination in adults with systemic rheumatic diseases: results from the COVID-19 Global Rheumatology Alliance Vaccine Survey. RMD Open 7(3)

58. Furer V, Eviatar T, Zisman D et al (2021) Immunogenicity and safety of the BNT162b2 mRNA COVID-19 vaccine in adult patients with autoimmune inflammatory rheumatic diseases and in the general population: a multicentre study. Ann Rheum Dis 80(10):1330-1338

Publisher's Note Springer Nature remains neutral with regard to jurisdictional claims in published maps and institutional affiliations.

\section{Authors and Affiliations}

\section{Michèle Keller $^{1} \cdot$ Laure F. Pittet $^{2,3,4}$ (D) $\cdot$ Petra Zimmermann ${ }^{1,3,5}$ (D)}

Michèle Keller

michele.keller@unifr.ch

Laure F. Pittet

laure.pittet@mcri.edu.au

1 Faculty of Science and Medicine, University of Fribourg, Fribourg, Switzerland

2 Pediatric Infectious Diseases Unit, Division of General Pediatrics, Department of Pediatrics, Gynecology \& Obstetrics, Faculty of Medicine, University Hospitals of Geneva and University of Geneva's, Geneva, Switzerland
3 Infectious Diseases Group, Murdoch Children's Research Institute, Parkville, VIC, Australia

4 Department of Paediatrics, The University of Melbourne, Parkville, VIC, Australia

5 Department of Paediatrics, Fribourg Hospital HFR, Fribourg, Switzerland 